\title{
Sharp IV bounds on average treatment effects on the treated and other populations under endogeneity and noncompliance
}

\author{
Martin Huber ${ }^{1}$, Lukas Laffers ${ }^{2}$, and Giovanni Mellace ${ }^{3}$ \\ ${ }^{1}$ University of Fribourg, Dept. of Economics, ${ }^{2}$ Matej Bel University, Dept. of Mathematics, \\ ${ }^{3}$ University of Southern Denmark, Dept. of Business and Economics
}

\begin{abstract}
In the presence of an endogenous binary treatment and a valid binary instrument, causal effects are point identified only for the subpopulation of compliers, given that the treatment is monotone in the instrument. With the exception of the entire population, causal inference for further subpopulations has been widely ignored in econometrics. We invoke treatment monotonicity and/or dominance assumptions to derive sharp bounds on the average treatment effects on the treated, as well as on other groups. Furthermore, we use our methods to assess the educational impact of a school voucher program in Colombia and discuss testable implications of our assumptions.
\end{abstract}

Keywords: instrument, noncompliance, principal stratification, nonparametric bounds.

We have benefited from comments by Sylvain Chabé-Ferret, Philipp Denter, Michael Lechner, Fabbrizia Mealli, Christoph Rothe, Anthony Strittmatter, seminar/conference participants in St. Gallen (research seminar), Tor Vergata (lunch seminar), Engelberg (labor seminar 2011), and Vancouver (SOLE 2011), and four anonymous referees. Addresses for correspondence: Martin Huber (martin.huber@unifr.ch), Department of Economics, University of Fribourg, Bd. de Pérolles 90, CH-1700 Fribourg; Lukáš Lafférs (lukas.laffers@gmail.com), Department of Mathematics, Matej Bel University, Tajovského 40, SK-97411 Banská Bystrica; Giovanni Mellace (giome@sam.sdu.dk), Department of Business and Economics, University of Southern Denmark, Campusvej 55, DK-5230 Odense M. 


\section{Introduction}

Endogeneity of the (binary) treatment variable and noncompliance to the treatment assignment in randomized experiments are widespread phenomena in the evaluation of treatment effects, see for instance Bloom (1984). Given a valid instrumental variable (IV) that is randomly assigned and has no direct effect on the mean potential outcomes and (weakly) positive monotonicity of the treatment in the instrument, Imbens and Angrist (1994) (see also Angrist, Imbens, and Rubin, 1996) show that the average treatment effect (ATE) is only identified in the subpopulation of compliers. The latter correspond to those whose the treatment status is equal to (i.e. reacts on) the instrument if both the treatment and the instrument are binary.

Whether the LATE is a relevant parameter heavily depends on the empirical context and has been controversially discussed in the literature, see for instance Imbens (2009), Deaton (2010), and Heckman and Urzúa (2010). Typically, researchers would like to identify the ATEs on the treated or the entire population. Note that these parameters are themselves weighted averages of the ATEs on several subpopulations, including the always takers (always treated irrespective of the instrument) and the never takers (never treated irrespective of the instrument). Maybe due to the fact that in a nonparametric framework, point identification is generally not feasible for the never takers, always takers, the treated, and the entire population (unless the complier share is $100 \%$ ), groups other than the compliers have (apart from the entire population) been widely ignored in the econometric literature. ${ }^{1}$

The main contribution of this paper is to derive nonparametric bounds on ATEs of populations that are potentially more policy relevant than the LATE on the compliers, which may not be externally valid. In particular, we also consider the treated population, which is of major interest in the program evaluation literature to assess the program effects on actual participants. In contrast to the commonly invoked full independence between the instrument and the potential outcomes/treatment states, we only assume mean independence between the instrument and the potential outcomes (within subpopulations) as well as the subpopulations. Moreover, we discuss

\footnotetext{
${ }^{1}$ An exception is Frölich and Lechner (2010) who also point identify the ATEs on the always takers and never takers. To this end, they invoke both IV and selection on observables (or conditional independence, see for instance Imbens, 2004) assumptions. However, this identification strategy stands in contrast to virtually all other IV applications, where an instrument is used exactly for the reason that no other source of identification (such as selection on observables) is available.
} 
the identifying power of (i) monotonicity of the treatment in the instrument and/or (ii) mean dominance of the potential outcomes of one subpopulation over the others. Monotonicity and dominance, either w.r.t. the mean or to the entire distribution (i.e., stochastic dominance), have also been considered in a different context, namely under non-random sample selection and attrition, see for instance Zhang and Rubin (2003), Lechner and Melly (2007), Blundell et al. (2007), Zhang et al. (2008), and Lee (2009), and Huber and Mellace (2013a). We use the principal stratification framework suggested by Frangakis and Rubin (2002) to derive sharp bounds for the ATEs on the always takers, never takers, the treated, the non-treated, and the entire population. ${ }^{2}$ As a further contribution, we find testable implications of the IV mean independence within subpopulations and mean dominance when monotonicity is invoked.

Partial identification of economic parameters in general goes back to Manski (1989, 1994) and Robins (1989). Previous work on nonparametric bounds under treatment endogeneity, which is the problem considered here, has almost exclusively focused on the ATE in the entire population, ${ }^{3}$ but neglected further populations. E.g., Manski (1990) bounds the ATE by solely relying on independence between the mean potential outcomes and the instrument. ${ }^{4}$ Considering binary outcomes, Balke and Pearl (1997) provide sharp bounds for the ATE under full (rather than mean) independence between the instrument and the potential values of the treatment (given the instrument) and the outcome (given the treatment) with and without monotonicity (see also Dawid, 2003) of the treatment in the instrument. Shaikh and Vytlacil (2011) bound the ATE on the entire population in the binary outcome case under monotonicity, too, and assume the treatment effect to be either weakly positive or weakly negative for all individuals (while the direction is a priori not restricted). See Bhattacharya et al. (2008) for an application. Cheng and Small (2006) extend the results for binary outcomes to three treatments (in contrast to the standard binary treatment framework considered here) under particular forms of (one-sided) noncompliance. Richardson and Robins (2010) is the only study apart from ours that also bounds the effects on further populations (compliers, defiers, never takers, and always takers). They assume full

\footnotetext{
${ }^{2}$ In addition, online Appendix A.8 provides the bounds for the treated subpopulations receiving and not receiving the instrument.

${ }^{3}$ For the derivation of semiparametric bounds on the ATE on the entire population, see Chiburis (2010) and the references therein.

${ }^{4} \mathrm{As}$ it is the aim of this paper to provide bounds for further populations, we also need to assume that the proportions of the subpopulations are independent of the instrument, otherwise the bounds on those populations might differ for different values of the instrument.
} 
independence and a binary outcome, but do not consider monotonicity or any form of mean dominance.

In contrast to much of the epidemiologic literature, Heckman and Vytlacil (2001) and Kitagawa (2009) allow for both discrete and continuous outcomes. Kitagawa (2009) partially identifies the potential outcome distributions for the entire population under (various forms of) full independence between the instrument and potential treatments/outcomes as well as monotonicity and derives bounds on the ATE. Also Heckman and Vytlacil (2001) assume full independence of the instrument, but invoke a nonparametric threshold crossing model characterizing the treatment choice instead of monotonicity for deriving the bounds on the ATE. However, by the results of Vytlacil (2002), both approaches are equivalent. One interesting finding of Heckman and Vytlacil (2001) and Kitagawa (2009) is that the width of their bounds is the same as those of Manski (1990), given that the monotonicity/threshold crossing model assumptions are satisfied. The present work adds to the literature on nonparametric bounds under endogeneity by considering more populations and an extended set of identifying assumptions than any of the previous studies.

The identifying power of monotonicity and mean dominance is demonstrated in an empirical application to Colombia's "Programa de Ampliación de Cobertura de la Educación Secundaria", which provided pupils from low income families with vouchers for private secondary schooling. Using experimental data previously analyzed by Angrist et al. (2002), we aim at assessing the program's impact on the educational achievement of various subpopulations. In particular, we find (in addition to the point identified complier effect) a significantly positive ATE on the treated which lies within reasonably tight bounds. This is an interesting result because it suggests that this and similar interventions have a positive effect on the participants, who are likely more policy relevant than the latent population of compliers.

The remainder of this paper is organized as follows. Section 2 characterizes the endogeneity/noncompliance problem based on principal stratification. Section 3 discusses the identifying assumptions and derives bounds on the ATEs for various populations. Section 4 briefly presents the estimators. In Section 5, we consider an empirical application to experimental education data. Section 6 concludes. 


\section{Using principal stratification to characterize non-}

\section{compliance}

Suppose that we want to estimate the effect of a binary treatment $D \in\{1,0\}$ (e.g., a training activity) on an outcome $Y$ (e.g., labor market success such as employment or earnings) evaluated at some point in time after the treatment. We use the experimental framework to motivate the problems of endogeneity and noncompliance. Assume that individuals are randomly assigned into treatment or non-treatment according to the binary assignment variable $Z \in\{1,0\}$, which will serve as instrument. Denote by $D_{i}(z)$ the potential treatment state for $Z=z$ and by $Y_{i}(d)$ the potential outcome (see for instance Rubin, 1974) of individual $i$ under treatment $D=d$. Throughout the discussion we will rule out interference between individuals or general equilibrium effects of the treatment by invoking the "Stable Unit Treatment Value assumption" (SUTVA), see for instance Rubin (1990). The SUTVA is formalized in Assumption 1:

\section{Assumption 1:}

$Y_{i}(d) \perp d_{j} \quad$ and $\quad D_{i}(z) \perp z_{j} \forall j \neq i, d \in\{0,1\}$ (SUTVA).

Even under Assumption 1, the individual effect $Y_{i}(1)-Y_{i}(0)$ can never be evaluated as individual $i$ is either treated or not treated, but cannot be observed in both states. I.e., the observed outcome $Y_{i}=D_{i} \cdot Y_{i}(1)+\left(1-D_{i}\right) \cdot Y_{i}(0)$. However, under particular assumptions aggregate parameters such as the average treatment effect (ATE) $\Delta=$ $E[Y(1)]-E[Y(0)]$ can be identified. E.g., assume that compliance in an experiment is perfect such that $D_{i}(1)=1$ and $D_{i}(0)=0$ for all individuals $i$. In this case and under successful randomization, $E[Y \mid Z=1]-E[Y \mid Z=0]=E[Y \mid D=1]-E[Y \mid D=0]=$ $E[Y(1)]-E[Y(0)]=\Delta$, where the first equality follows from perfect compliance and the second from random assignment. I.e., the ATE is identified because all individuals are compliers. However, if post-assignment complications occur such that $D_{i}(z) \neq z$ for some $z$ and some individuals $i$, selection bias may flaw the validity of the evaluation in spite of the randomization of the assignment. This is due to the potential threat that individuals systematically select themselves into the treatment according to their potential outcomes.

Using the principal stratification framework advocated by Frangakis and Rubin (2002), the population can be divided into four principal strata, denoted by $T$, ac- 
cording to the choice of $D$ as a reaction of $Z$. Angrist et al. (1996) refer to the four groups as (i) compliers, who react on the instrument in the intended way by taking the treatment when $Z=1$ and abstaining from it when $Z=0$, (ii) always takers, who are always treated irrespective of the assignment, (iii) never takers, who are never treated irrespective of the assignment, and (iv) defiers, who are treated when not assigned, but not treated when assigned. Table 1 visualizes this definition.

Table 1: Principal strata

\begin{tabular}{c|cc|c}
\hline \hline Principal strata $(T)$ & $\mathrm{D}(1)$ & $\mathrm{D}(0)$ & Notion \\
\hline 11 & 1 & 1 & Always takers \\
10 & 1 & 0 & Compliers \\
01 & 0 & 1 & Defiers \\
00 & 0 & 0 & Never takers \\
\hline
\end{tabular}

It is obvious that we cannot directly observe the principal stratum an individual belongs to as either $D(1)$ or $D(0)$ is known. Therefore, without the imposition of further assumptions, neither the principal strata proportions nor the distribution of $Y$ within any stratum are identified. To see this, note that the observed values of $Z$ and $D$ generate four observed subgroups which are all mixtures of two principal strata. This implies that any individual $i$ with a particular combination of $Z_{i}, D_{i}$ may belong to two principal strata, see Table 2 .

Table 2: Observed subgroups and principal strata

\begin{tabular}{l|c}
\hline \hline Observed subgroups & principal strata \\
\hline$\left\{i: Z_{i}=1, D_{i}=1\right\}$ & subject $i$ belongs either to 11 or to 10 \\
$\left\{i: Z_{i}=1, D_{i}=0\right\}$ & subject $i$ belongs either to 01 or to 00 \\
$\left\{i: Z_{i}=0, D_{i}=1\right\}$ & subject $i$ belongs either to 11 or to 01 \\
$\left\{i: Z_{i}=0, D_{i}=0\right\}$ & subject $i$ belongs either to 10 or to 00 \\
\hline
\end{tabular}

As second identifying restriction maintained throughout the paper, we will assume $Z$ to be independent of (i) the mean potential outcomes within principal strata and (ii) of the strata proportions, which has also been considered in Frölich (2007):

\section{Assumption 2:}

(i) $E(Y(d) \mid T=t, Z=1)=E(Y(d) \mid T=t, Z=0)=E(Y(d) \mid T=t)$ for $d \in\{0,1\}$ and $t \in\{11,10,01,00\} \quad$ (mean independence within principal strata),

(ii) $\operatorname{Pr}(T=t \mid Z=1)=\operatorname{Pr}(T=t \mid Z=0)=\operatorname{Pr}(T=t)$ for $t \in\{11,10,01,00\}$ (unconfounded strata proportions).

Assumption 2 (i) postulates that mere assignment does not have any direct effect on the mean potential outcomes within any stratum other than through the treatment, ${ }^{5}$

\footnotetext{
${ }^{5}$ However, in contrast to the full independence considered in Imbens and Angrist (1994) and Angrist
} 
i.e. mean independence within principal strata. ${ }^{6}$ Taking assignment to a training as an example, it rules out that the average labor market success given $T$ changes as a reaction to merely being assigned. I.e., what should matter is whether the training is actually received. By Assumption 2 (ii), the proportion of any stratum conditional on the instrument is equal to its unconditional proportion in the entire population. This holds for instance under random assignment, where $Z$ is fully independent of the joint distribution of $(D(1), D(0))$ and thus, of $T$. Alternatively to the unconditional validity of Assumption 2, one may assume that it only holds conditional on some observed preassignment variables $X$. This is closely related to the framework of Frölich (2007) who shows point identification of the LATE under a conditionally valid instrument (given $X$ ). In the further discussion, conditioning on $X$ will be kept implicit, such that all results either refer to the experimental framework or to an analysis within cells of $X$.

Unfortunately, even under Assumptions 1 and 2, point identification is not obtained. Let $\pi_{t}=\operatorname{Pr}(T=t)$ denote a particular proportion and $P_{d \mid z} \equiv \operatorname{Pr}(D=$ $d \mid Z=z)$ the observed treatment probability conditional on assignment status. Under Assumption 2 (ii), which ensures that the strata proportions conditional on the instrument are equal to the unconditional strata proportions, the relation between the observed $P_{d \mid z}$ and the latent $\pi_{t}$ is as displayed in Table 3. Likewise, any observed

Table 3: Observed probabilities and principal strata proportions

\begin{tabular}{c|c}
\hline \hline Observed cond. treatment prob. & princ. strata proportions \\
\hline$P_{1 \mid 1}=\operatorname{Pr}(D=1 \mid Z=1)$ & $\pi_{11}+\pi_{10}$ \\
$P_{0 \mid 1}=\operatorname{Pr}(D=0 \mid Z=1)$ & $\pi_{01}+\pi_{00}$ \\
$P_{1 \mid 0}=\operatorname{Pr}(D=1 \mid Z=0)$ & $\pi_{11}+\pi_{01}$ \\
$P_{0 \mid 0}=\operatorname{Pr}(D=0 \mid Z=0)$ & $\pi_{10}+\pi_{00}$ \\
\hline
\end{tabular}

conditional mean outcome is a mixture of the mean outcomes of two strata. E.g.,

$$
\begin{aligned}
E(Y \mid Z=1, D=1) & =\frac{\pi_{11}}{\pi_{11}+\pi_{10}} \cdot E(Y \mid Z=1, D=1, T=11) \\
& +\frac{\pi_{10}}{\pi_{11}+\pi_{10}} \cdot E(Y \mid Z=1, D=1, T=10), \\
& =\frac{\pi_{11}}{\pi_{11}+\pi_{10}} \cdot E(Y \mid D=1, T=11)+\frac{\pi_{10}}{\pi_{11}+\pi_{10}} \cdot E(Y \mid D=1, T=10), \\
& =\frac{\pi_{11}}{\pi_{11}+\pi_{10}} \cdot E(Y(1) \mid T=11)+\frac{\pi_{10}}{\pi_{11}+\pi_{10}} \cdot E(Y(1) \mid T=10),
\end{aligned}
$$

where the second equality follows from Assumption 2 (i) and the third from the fact

et al. (1996), it may affect higher moments.

${ }^{6}$ For the case that mean independence is not satisfied, Flores and Flores-Lagunes (2013) derive bounds on the LATE in the presence of an invalid instrument. In contrast, we will assume the instrument to be valid (in the sense that it satisfies Assumption 2) throughout the discussion. 
that the treatment is unconfounded within each stratum consisting of individuals with identical (non-)compliance behavior.

Thus, point identification of causal effects would require us to invoke further assumptions. E.g., under monotonicity of $D$ in $Z$ and effect homogeneity, the ATE on the entire population is identified. Albeit used in much of the IV literature, effect homogeneity is a very unattractive assumption given the rich empirical evidence on effect heterogeneity in the field of treatment evaluation. Under monotonicity and effect heterogeneity, the LATE on the compliers is identified, but this effect may be "too local" to be of policy interest. Fortunately, assumptions as monotonicity and mean dominance also bear identifying power for further populations and may yield informative bounds, as discussed in the next section.

\section{Assumptions and interval identification}

\subsection{Mean independence within principal strata without fur- ther assumptions}

The partial identification of ATEs on various populations will be based on bounding the mean potential outcomes $E(Y(1) \mid T=t), E(Y(0) \mid T=t)$, with $t \in\{11,10,00,01\}$. To this end, we assume that the support $\mathcal{Y}$ of the outcome variable $Y$ is bounded, i.e., $\mathcal{Y}=\left[y^{L B}, y^{U B}\right]$ with $-\infty<y^{L B}<y^{U B}<\infty$, and that $Y$ is continuous over $\mathcal{Y}$ (see online Appendix A.5 for discrete outcomes). Boundedness of $Y$ rules out infinite upper or lower bounds on the mean potential outcomes and thus, on the ATE in any population. We will refer to the bounds on any ATE as being informative if its identification region does not coincide with (i.e. is tighter than) $\left[y^{L B}-y^{U B}, y^{U B}-y^{L B}\right]$.

Partial identification is obtained in three steps. In the first step, we derive sharp bounds on the principal strata proportions using Assumption 2 (ii). As one can express three out of four proportions as a function of the remaining one, we only need to bound the latter. Therefore, all bounds are computed as functions of the defier proportion, but choosing any other principal stratum would entail the same results. The second step (which is mostly discussed in the online appendix) gives the bounds on the mean potential outcomes and the ATEs conditional on the defier proportion. It makes use of the fact that each observed conditional mean outcome is a mixture of the mean potential outcomes of two principal strata, with the mixing probabilities 
corresponding to the relative principal strata proportions:

$$
\begin{aligned}
& E(Y \mid Z=1, D=1)=\frac{\pi_{11}}{\pi_{11}+\pi_{10}} \cdot E(Y(1) \mid T=11)+\frac{\pi_{10}}{\pi_{11}+\pi_{10}} \cdot E(Y(1) \mid T=1001,) \\
& E(Y \mid Z=0, D=1)=\frac{\pi_{11}}{\pi_{11}+\pi_{01}} \cdot E(Y(1) \mid T=11)+\frac{\pi_{01}}{\pi_{11}+\pi_{01}} \cdot E(Y(1) \mid T=01(2) \\
& E(Y \mid Z=0, D=0)=\frac{\pi_{10}}{\pi_{00}+\pi_{10}} \cdot E(Y(0) \mid T=10)+\frac{\pi_{00}}{\pi_{00}+\pi_{10}} \cdot E(Y(0) \mid T=0003) \\
& E(Y \mid Z=1, D=0)=\frac{\pi_{01}}{\pi_{00}+\pi_{01}} \cdot E(Y(0) \mid T=01)+\frac{\pi_{00}}{\pi_{00}+\pi_{01}} \cdot E(Y(0) \mid T=0004)
\end{aligned}
$$

Given the defier proportion (and thus, the mixing probabilities), the results of Horowitz and Manski (1995) (see Section 3.2 and Proposition 4 therein) provide us with sharp bounds on the mean potential outcomes within each of equations (1) to (4), whereas Assumption 2 (i) allows further tightening these bounds across equations. Using an approach inspired by Kitagawa (2009), we derive sharp bounds on the mean potential outcomes and the ATEs under Assumption 2 (i) and conditional on the defier proportion. Finally, taking the supremum (infimum) of the ATEs in the second step over admissible defier proportions that satisfy Assumption 2 (ii) yields the sharp upper (lower) bounds on the ATEs (see online Appendix A.1.2). ${ }^{7}$ As the bounds are continuous in $\pi_{01}$ (as shown in online Appendix A.1.4) and the set of admissible shares of defiers is an interval (as shown in Lemma 1), the optima are attained by the extreme value theorem.

Concerning the bounds on the defier proportion, note that under Assumptions 1 and 2, Table 3 provides us with the following equations:

$$
\begin{array}{cl}
\pi_{11}=P_{1 \mid 0}-\pi_{01} & \Rightarrow \pi_{01} \leq P_{1 \mid 0}, \\
\pi_{00}=P_{0 \mid 1}-\pi_{01} & \Rightarrow \pi_{01} \leq P_{0 \mid 1}, \\
\pi_{10}=P_{1 \mid 1}-P_{1 \mid 0}+\pi_{01} \quad & \Rightarrow \pi_{01} \geq P_{1 \mid 0}-P_{1 \mid 1},
\end{array}
$$

and thus, the defier proportion must lie in the following set

$$
\pi_{01} \in \mathcal{P}=\left[\max \left(0, P_{1 \mid 0}-P_{1 \mid 1}\right), \min \left(P_{1 \mid 0}, P_{0 \mid 1}\right)\right]
$$

Note that these bounds are valid outer bounds, but they need not be sharp. Sharp bounds on the proportion of defiers have to be constructed based on the joint dis-

\footnotetext{
${ }^{7}$ It is worth noting that without further restrictions, it is generally not possible that one particular value of $\pi_{01}$ jointly optimizes the bounds on all ATEs considered.
} 
tribution of $(Y, D, Z)$, rather than $(D, Z)$ alone. Under Assumptions 1 and 2, the condition $\pi_{01}=0$ leads to testable implications as studied in Huber and Mellace (2013b). However, in some cases zero may not be in the identified set of the defier share. In online Appendix A.1.3, we show that admissibility of $\pi_{01}$ is equivalent to checking four moment inequalites that generalize those inequalities outlined in $\mathrm{Hu}$ ber and Mellace (2013b). Furthermore, online Appendix A.1.1 presents a linear programming procedure for constructing sharp bounds on $\pi_{01}$ in the case of a discrete $Y{ }^{8}$ Under discreteness, linear programming can also be used for constructing sharp bounds on various types of ATEs as it was first pointed out by Balke and Pearl (1997). This work was further extended to the case with continuous outcomes by Kitagawa (2009), who derived a closed-form solution for the sharp bounds (rather than relying on linear programming). Lafférs (2013) applied linear programming to bound ATEs under various sets of assumptions.

We denote by $\pi_{t}^{\min }$ and $\pi_{t}^{\max }$ the sharp lower and upper bounds of $\pi_{t}, t=$ $11,10,01,00$, respectively, and by $\mathcal{P}^{*}$ the sharp identified set for $\pi_{01}$ under Assumptions 1 and 2. In online Appendix A.1.1, we show that $\mathcal{P}^{*}$ is an interval. If $\mathcal{P}=\mathcal{P}^{*}$, then the remaining strata proportions can be bounded by substituting (6) into (5). It is easy to see that either $\pi_{01}^{\min }=0$ or $\pi_{10}^{\min }=0$ and either $\pi_{11}^{\min }=0$ or $\pi_{00}^{\min }=0$. The outer bounds $\mathcal{P}$ are equivalent to those derived in Richardson and Robins (2010) (equation (6) of Section 3.1, page 9). In contrast to their paper we only assume mean independence within principal strata in Assumption 2, rather than full independence.

In order to bound the ATEs on the four populations, we introduce some additional notation. We define $\bar{Y}_{z, d}=E(Y \mid Z=z, D=d)$ to be the conditional mean of $Y$ given $Z=z$ and $D=d$. Furthermore, $F_{Y_{z, d}}(y)=\operatorname{Pr}(Y \leq y \mid Z=z, D=d)$ denotes the conditional cdf of $Y$ given $Z=z$ and $D=d$. Let $q_{z, d}^{t}$ denote the share of individuals belonging to stratum $T=t$ in the observed subgroup with $Z=z$ and $D=d$. If necessary, we will denote by $q_{z, d}^{t, \pi_{01}^{\max }}$ and $q_{z, d}^{t, \pi_{01}^{\min }}$, the value of $q_{z, d}^{t}$ when $\pi_{01}$ is equal to $\pi_{01}^{\max }$ or $\pi_{01}^{\min }$, respectively. Let $F_{Y_{z, d}}^{-1}\left(q_{z, d}^{t}\right)=\inf \left\{y: F_{Y_{z, d}}(y) \geq q_{z, d}^{t}\right\}^{9}$ be the conditional quantile function of $\mathrm{Y}$ given $Z=z$ and $D=d$. We can then define the lower and upper bounds of $E(Y \mid Z=z, D=d, T=t)$, which by Assumption 2 corresponds to $E(Y(d) \mid T=$ t) (see Section 2), as $\bar{Y}_{z, d}\left(\min \mid q_{z, d}^{t}\right)=E\left(Y \mid Z=z, D=d, Y \leq F_{Y_{z, d}}^{-1}\left(q_{z, d}^{t}\right)\right)$ and

\footnotetext{
${ }^{8}$ We thank an anonymous referee for pointing out the difference between $\mathcal{P}$ and $\mathcal{P}^{*}$ and for suggesting the linear programming tool.

${ }^{9}$ We define $F_{Y_{z, d}}^{-1}(0) \equiv y^{L B}$ and $F_{Y_{z, d}}^{-1}(1) \equiv y^{U B}$.
} 
$\bar{Y}_{z, d}\left(\max \mid q_{z, d}^{t}\right) \equiv E\left(Y \mid Z=z, D=d, Y \geq F_{Y_{z, d}}^{-1}\left(1-q_{z, d}^{t}\right)\right)$, respectively. Finally, the ATEs on the various principal strata, the treated, the non-treated, and the entire population are denoted by $\Delta_{t} \equiv E(Y(1)-Y(0) \mid T=t)$ with $t \in\{11,10,01,00\}$, $\Delta_{D=d} \equiv E(Y(1)-Y(0) \mid D=d)$ with $d \in\{1,0\}$, and $\Delta \equiv E(Y(1)-Y(0))$, respectively. The superscripts "UB" and "LB" denote the sharp upper and lower bounds on the respective parameters, where sharpness of the bounds on some parameter $\tilde{\Delta}$ is defined as follows:

Definition 1 Given the knowledge of the distribution of the observed data, $\tilde{\Delta}^{L B}$ and $\tilde{\Delta}^{U B}$ are sharp if $\left[\tilde{\Delta}^{L B}, \tilde{\Delta}^{U B}\right]$ is the shortest interval such that, for every $\tilde{\Delta} \in$ $\left[\tilde{\Delta}^{L B}, \tilde{\Delta}^{U B}\right]$, we can construct principal strata proportions $\operatorname{Pr}(T \mid Z): T=11,10,01,00$, $Z=1,0$ and potential outcome distributions $f(Y(1), Y(0) \mid T, Z): T=11,10,01,00$, $Z=1,0$ that satisfy the imposed assumptions.

Considering the ATE on the compliers $\left(\Delta_{10}\right)$, if $P_{1 \mid 0}-P_{1 \mid 1} \notin \mathcal{P}^{*}$, then the upper and lower bounds are, respectively,

$$
\begin{aligned}
\Delta_{10}^{U B} & =\sup _{\pi_{01} \in \mathcal{P}^{*}}\left[\frac{P_{1 \mid 1} \cdot \bar{Y}_{1,1}-\left(P_{1 \mid 0}-\pi_{01}\right) \cdot \max \left(\bar{Y}_{1,1}\left(\min \mid q_{1,1}^{11}\right), \bar{Y}_{0,1}\left(\min \mid q_{0,1}^{11}\right)\right)}{P_{1 \mid 1}-P_{1 \mid 0}+\pi_{01}}\right. \\
& \left.-\frac{P_{0 \mid 0} \cdot \bar{Y}_{0,0}-\left(P_{0 \mid 1}-\pi_{01}\right) \cdot \min \left(\bar{Y}_{0,0}\left(\max \mid q_{0,0}^{00}\right), \bar{Y}_{1,0}\left(\max \mid q_{1,0}^{00}\right)\right)}{P_{1 \mid 1}-P_{1 \mid 0}+\pi_{01}}\right], \\
\Delta_{10}^{L B} & =\inf _{\pi_{01} \in \mathcal{P}^{*}}\left[\frac{P_{1 \mid 1} \cdot \bar{Y}_{1,1}-\left(P_{1 \mid 0}-\pi_{01}\right) \cdot \min \left(\bar{Y}_{1,1}\left(\max \mid q_{1,1}^{11}\right), \bar{Y}_{0,1}\left(\max \mid q_{0,1}^{11}\right)\right)}{P_{1 \mid 1}-P_{1 \mid 0}+\pi_{01}}\right. \\
& \left.-\frac{P_{0 \mid 0} \cdot \bar{Y}_{0,0}-\left(P_{0 \mid 1}-\pi_{01}\right) \cdot \max \left(\bar{Y}_{0,0}\left(\min \mid q_{0,0}^{00}\right), \bar{Y}_{1,0}\left(\min \mid q_{1,0}^{00}\right)\right)}{P_{1 \mid 1}-P_{1 \mid 0}+\pi_{01}}\right],
\end{aligned}
$$

where $q_{1,1}^{11}=\frac{P_{1 \mid 0}-\pi_{01}}{P_{1 \mid 1}}$ (the share of always takers among those with $Z=1$ and $D=1$ ), $q_{0,1}^{11}=\frac{P_{1 \mid 0}-\pi_{01}}{P_{1 \mid 0}}$ (the share of always takers among those with $Z=0$ and $D=1$ ), $q_{1,0}^{00}=\frac{P_{0 \mid 1}-\pi_{01}}{P_{0 \mid 1}}$ (the share of never takers among those with $Z=0$ and $D=1$ ), and $q_{0,0}^{00}=\frac{P_{0 \mid 1}-\pi_{01}}{P_{0 \mid 0}}$ (the share of never takers among those with $Z=0$ and $D=0$ ). The proofs of the sharpness of these bounds as well as of any other bounds proposed below are provided in the online appendix. If $\pi_{01}=P_{1 \mid 0}-P_{1 \mid 1} \in \mathcal{P}^{*}$, then $\pi_{10}=0$ and the bounds are uninformative. Therefore, $\Delta_{10}^{U B}=y^{U B}-y^{L B}$ and $\Delta_{10}^{L B}=y^{L B}-y^{U B}$. In online Appendix A.1.4, we show that $\Delta^{U B}\left(\pi_{01}\right)$ and $\Delta^{L B}\left(\pi_{01}\right)$ are continuous in $\pi_{01}$.

Four points are worth noting concerning the derivation of these bounds. Firstly and as already mentioned, they make use of Proposition 4 of Horowitz and Manski (1995), which in general only holds for continuous outcomes. Fortunately, it is easy to 
show that their results can also be applied to discrete outcomes after a modification of the trimming function, see online Appendix A.5 for further details. Secondly, (7) has to be optimized w.r.t. admissible defier proportions, given by $\mathcal{P}^{*}$. Thirdly, mean independence within strata (Assumption 2 (i)) gives rise to the maximum and minimum operators. Note that in the first (third) line in (7) one computes the upper (lower) bound of the compliers' mean potential outcome under treatment by subtracting the lower (upper) bound of the mean potential outcome of the always takers. As their lower (upper) bound under treatment is not affected by the value of $Z$ due to mean independence, the lower (upper) bound is the maximum (minimum) of the always takers' lower (upper) bounds for $Z=1$ and $Z=0$. An analogous result holds for lines 2 and 4 w.r.t. the bounds on the potential mean outcomes under non-treatment of the never takers. Finally, if $\mathcal{P}=\mathcal{P}^{*}$, these bounds are informative only if $P_{1 \mid 0}<P_{1 \mid 1}$. This is equivalent to $\pi_{10}>\pi_{01}$, saying that the share of compliers is larger than the share of defiers. The reason is that if $P_{1 \mid 0} \geq P_{1 \mid 1}$, then $\pi_{01}^{\min }=P_{1 \mid 0}-P_{1 \mid 1}>0$, which implies that $\pi_{10}^{\min }=0$ (so that the non-existence of compliers cannot be ruled out) and $\Delta_{10}^{U B}=y^{U B}-y^{L B}, \Delta_{10}^{L B}=y^{U B}-y^{L B}$.

In a symmetric way one obtains the sharp upper and lower bounds on the ATE on the defiers, $\Delta_{01}$. If $0 \notin \mathcal{P}^{*}$ :

$$
\begin{aligned}
\Delta_{01}^{U B} & =\sup _{\pi_{01} \in \mathcal{P}^{*}}\left[\frac{P_{1 \mid 0} \cdot \bar{Y}_{0,1}-\left(P_{1 \mid 0}-\pi_{01}\right) \cdot \max \left(\bar{Y}_{1,1}\left(\min \mid q_{1,1}^{11}\right), \bar{Y}_{0,1}\left(\min \mid q_{0,1}^{11}\right)\right)}{\pi_{01}}\right. \\
& \left.-\frac{P_{0 \mid 1} \cdot \bar{Y}_{1,0}-\left(P_{0 \mid 1}-\pi_{01}\right) \cdot \min \left(\bar{Y}_{0,0}\left(\max \mid q_{0,0}^{00}\right), \bar{Y}_{1,0}\left(\max \mid q_{1,0}^{00}\right)\right)}{\pi_{01}}\right], \\
\Delta_{01}^{L B} & =\inf _{\pi_{01} \in \mathcal{P}^{*}}\left[\frac{P_{1 \mid 0} \cdot \bar{Y}_{0,1}-\left(P_{1 \mid 0}-\pi_{01}\right) \cdot \min \left(\bar{Y}_{1,1}\left(\max \mid q_{1,1}^{11}\right), \bar{Y}_{0,1}\left(\max \mid q_{0,1}^{11}\right)\right)}{\pi_{01}}\right. \\
& \left.-\frac{P_{0 \mid 1} \cdot \bar{Y}_{1,0}-\left(P_{0 \mid 1}-\pi_{01}\right) \cdot \max \left(\bar{Y}_{0,0}\left(\min \mid q_{0,0}^{00}\right), \bar{Y}_{1,0}\left(\min \mid q_{1,0}^{00}\right)\right)}{\pi_{01}}\right],
\end{aligned}
$$

For the same reason mentioned above, if $\mathcal{P}=\mathcal{P}^{*}$, these bounds are informative only if $P_{1 \mid 0}>P_{1 \mid 1}$, i.e., if there are more defiers than compliers. Therefore, without imposing further assumptions, the bounds are informative either for the defiers or for the compliers, but never for both populations. Furthermore, unless either $P_{1 \mid 1}-P_{1 \mid 0}=$ 0 or $\mathcal{P} \neq \mathcal{P}^{*}$, either positive (if $P_{1 \mid 1}-P_{1 \mid 0}>0$ ) or negative (if $P_{1 \mid 0}-P_{1 \mid 1}>0$ ) monotonicity of $D$ in $Z$ can be consistent with the data, but not both at the same time.

Concerning the always takers, note that their outcomes are only observed under 
treatment. The shares of always takers in the observed groups with $Z=1, D=1$ and $Z=0, D=1$ are, respectively, $\pi_{11} /\left(\pi_{11}+\pi_{10}\right)=\left(P_{1 \mid 0}-\pi_{01}\right) / P_{1 \mid 1}$ and $\pi_{11} /\left(\pi_{11}+\right.$ $\left.\pi_{01}\right)=\left(P_{1 \mid 0}-\pi_{01}\right) / P_{1 \mid 0}$. Therefore, we can bound the upper and lower values of the mean potential outcome under treatment for this population by $\min \left(\bar{Y}_{1,1}\left(\max \mid q_{1,1}^{11, \pi_{01}^{\max }}\right), \bar{Y}_{0,1}\left(\max \mid q_{0,1}^{11, \pi_{01}^{\max }}\right)\right)$ and $\max \left(\bar{Y}_{1,1}\left(\min \mid q_{1,1}^{11, \pi_{01}^{\max }}\right), \bar{Y}_{0,1}\left(\min \mid q_{0,1}^{11, \pi_{01}^{\max }}\right)\right)$, respectively. As already discussed, the intuition for the optimization over different values of the instrument is that $Z$ does not have a direct effect on the mean potential outcomes. Therefore, the set of admissible potential outcomes for $D=1$ is the intersection of possible values under $Z=0$ and $Z=1$.

Since the outcomes of the always takers are never observed under non-treatment, we have to rely on the upper and lower bounds in the support of $Y, y^{U B}$ and $y^{L B}$. The sharp upper and lower bounds for the ATE on the always takers $\Delta_{11}$, are:

$$
\begin{aligned}
& \Delta_{11}^{U B}=\min \left(\bar{Y}_{1,1}\left(\max \mid q_{1,1}^{11, \pi_{01}^{\max }}\right), \bar{Y}_{0,1}\left(\max \mid q_{0,1}^{11, \pi_{01}^{\max }}\right)\right)-y^{L B}, \\
& \Delta_{11}^{L B}=\max \left(\bar{Y}_{1,1}\left(\min \mid q_{1,1}^{11, \pi_{01}^{\max }}\right), \bar{Y}_{0,1}\left(\min \mid q_{0,1}^{11, \pi_{01}^{\max }}\right)\right)-y^{U B} .
\end{aligned}
$$

It is easy to see ${ }^{10}$ that $\pi_{01}^{\max }$ maximizes the upper bound and minimizes the lower bound of $\Delta_{11}$ w.r.t. $\pi_{01}$, so that $q_{z, 1}^{11, \pi_{01}^{\max }}=\max \left(0, \frac{P_{1 \mid 0}-P_{0 \mid 1}}{P_{1 \mid z}}\right)$. Similarly as for the compliers and defiers, these bounds are only informative if $P_{1 \mid 0}>P_{0 \mid 1} \Rightarrow \pi_{11}>\pi_{00}$, i.e., if the share of always takers is larger than the share of never takers. The sampling process constraints the identification region of either the average treatment effect on the always takers or of the one on the never takers. Once again if $P_{1 \mid 0}<P_{0 \mid 1}$ then $\pi_{01}^{\max }=P_{1 \mid 0}$, which implies that $\pi_{11}^{\min }=0$.

Using a symmetric argument as for the always takers, the sharp upper and lower bounds on the ATE of the never takers, $\Delta_{00}$, are, respectively:

$$
\begin{aligned}
& \Delta_{00}^{U B}=y^{U B}-\max \left(\bar{Y}_{1,0}\left(\min \mid q_{1,0}^{00, \pi_{01}^{\max }}\right), \bar{Y}_{0,0}\left(\min \mid q_{0,0}^{00, \pi_{01}^{\max }}\right)\right), \\
& \Delta_{00}^{L B}=y^{L B}-\min \left(\bar{Y}_{1,0}\left(\max \mid q_{1,0}^{00, \pi_{01}^{\max }}\right), \bar{Y}_{0,0}\left(\max \mid q_{0,0}^{00, \pi_{01}^{\max }}\right)\right) .
\end{aligned}
$$

$\pi_{01}^{\max }$ maximizes the upper bound and minimizes the lower bound of $\Delta_{00}$ w.r.t. $\pi_{01}$, such that $q_{z, 0}^{00, \pi_{01}^{\max }}=\max \left(0, \frac{P_{0 \mid 1}-P_{1 \mid 0}}{P_{0 \mid z}}\right)$. The bounds are informative if $P_{1 \mid 0}<P_{0 \mid 1}$,

\footnotetext{
${ }^{10}$ Both $\bar{Y}_{1,1}\left(\max \mid q_{1,1}^{11}\right)$ and $\bar{Y}_{0,1}\left(\max \mid q_{0,1}^{11}\right)$ are non-decreasing functions of $\pi_{01}$. Similarly, both $\bar{Y}_{1,1}\left(\min \mid q_{1,1}^{11}\right)$ and $\bar{Y}_{0,1}\left(\min \mid q_{0,1}^{11}\right)$ are non-increasing functions of $\pi_{01}$.
} 
i.e., if there are more never takers than always takers in the population. Similar as before if $P_{1 \mid 0}>P_{0 \mid 1}, \pi_{01}^{\max }=P_{0 \mid 1}$ which implies that $\pi_{00}^{\min }=0 .{ }^{11}$

The identification results presented so far refer to latent strata defined by $Z$ and $D$, populations that are not directly observed in the data. However, in the program evaluation literature, most attention seems to be devoted to the (observed) population receiving the treatment, see for instance Heckman et al. (1999), which generally appears more policy relevant than latent groups. As a major contribution of this paper, we therefore also derive sharp bounds on the ATEs on the treated, as well as the nontreated and the entire population. The discussion below shows that for doing so, it suffices to establish the sharp bounds on $E(Y(1))$ and $E(Y(0))$, which for continuous outcomes are given by

$$
\begin{aligned}
E(Y(1))^{U B} & =\left(P_{0 \mid 1}-\pi_{01}^{\min }\right) \cdot y^{U B}-\left(P_{1 \mid 0}-\pi_{01}^{\min }\right) \cdot \max \left(\bar{Y}_{1,1}\left(\min \mid q_{1,1}^{11, \pi_{01}^{\min }}\right), \bar{Y}_{0,1}\left(\min \mid q_{0,1}^{11, \pi_{01}^{\min }}\right)\right) \\
+ & P_{1 \mid 0} \cdot \bar{Y}_{0,1}+P_{1 \mid 1} \cdot \bar{Y}_{1,1}, \\
E(Y(1))^{L B} & =\left(P_{0 \mid 1}-\pi_{01}^{\min }\right) \cdot y^{L B}-\left(P_{1 \mid 0}-\pi_{01}^{\min }\right) \cdot \min \left(\bar{Y}_{1,1}\left(\max \mid q_{1,1}^{11, \pi_{01}^{\min }}\right), \bar{Y}_{0,1}\left(\max \mid q_{0,1}^{11, \pi_{01}^{\min }}\right)\right) \\
+ & P_{1 \mid 0} \cdot \bar{Y}_{0,1}+P_{1 \mid 1} \cdot \bar{Y}_{1,1},
\end{aligned}
$$

and

$$
\begin{aligned}
E(Y(0))^{U B} & =\left(P_{1 \mid 0}-\pi_{01}^{\min }\right) \cdot y^{U B}-\left(P_{0 \mid 1}-\pi_{01}^{\min }\right) \cdot \max \left(\bar{Y}_{0,0}\left(\min \mid q_{0,0}^{00, \pi_{01}^{\min }}\right), \bar{Y}_{1,0}\left(\min \mid q_{1,0}^{00, \pi_{01}^{\min }}\right)\right) \\
+ & P_{0 \mid 0} \cdot \bar{Y}_{0,0}+P_{0 \mid 1} \cdot \bar{Y}_{1,0}, \\
E(Y(0))^{L B} & =\left(P_{1 \mid 0}-\pi_{01}^{\min }\right) \cdot y^{L B}-\left(P_{0 \mid 1}-\pi_{01}^{\min }\right) \cdot \min \left(\bar{Y}_{0,0}\left(\max \mid q_{0,0}^{00, \pi_{01}^{\min }}\right), \bar{Y}_{1,0}\left(\max \mid q_{1,0}^{00, \pi_{01}^{\min }}\right)\right) \\
& +P_{0 \mid 0} \cdot \bar{Y}_{0,0}+P_{0 \mid 1} \cdot \bar{Y}_{1,0},
\end{aligned}
$$

respectively, see online Appendix A.1.5. If $Y$ is not continuous these bounds, and thus the one on the treated, non-treated, and the entire population, have to be optimized w.r.t. admissible defier proportions $\pi_{01}$, given by $\mathcal{P}^{*}$.

Considering the ATE on the treated, $\Delta_{D=1}=E(Y(1)-Y(0) \mid D=1)$, note that because $E(Y(1) \mid D=1)=E(Y \mid D=1)$ is identified from the data, we only need to bound $E(Y(0) \mid D=1)$. Solving $E(Y(0))=\operatorname{Pr}(D=1) \cdot E(Y(0) \mid D=1)+\operatorname{Pr}(D=0)$. $E(Y(0) \mid D=0)$ for $E(Y(0) \mid D=1)$ gives $E(Y(0) \mid D=1)=\frac{E(Y(0))-\operatorname{Pr}(D=0) \cdot E(Y \mid D=0)}{\operatorname{Pr}(D=1)}$.

\footnotetext{
${ }^{11}$ This demonstrates that it is generally not possible to have a value of $\pi_{01}$ that jointly optimizes the bounds on the average treatment effects of all principal strata.
} 
Letting $E(Y(0))^{U B}$ and $E(Y(0))^{L B}$ denote the sharp upper and lower bounds for $E(Y(0))$, respectively, it therefore follows that the sharp upper and lower bounds on the ATE on the treated $\Delta_{D=1}$ are given by

$$
\begin{aligned}
& \Delta_{D=1}^{U B}=E(Y \mid D=1)-\frac{E(Y(0))^{L B}-\operatorname{Pr}(D=0) \cdot E(Y \mid D=0)}{\operatorname{Pr}(D=1)}, \\
& \Delta_{D=1}^{L B}=E(Y \mid D=1)-\frac{E(Y(0))^{U B}-\operatorname{Pr}(D=0) \cdot E(Y \mid D=0)}{\operatorname{Pr}(D=1)} .
\end{aligned}
$$

Since

$$
\operatorname{Pr}(D=0) \cdot E(Y \mid D=0)=\operatorname{Pr}(Z=0) \cdot P_{0 \mid 0} \cdot \bar{Y}_{0,0}+\operatorname{Pr}(Z=1) \cdot P_{0 \mid 1} \cdot \bar{Y}_{1,0}
$$

and

$$
\begin{aligned}
P_{0 \mid 0} \cdot \bar{Y}_{0,0}+P_{0 \mid 1} \cdot \bar{Y}_{1,0} & =\operatorname{Pr}(Z=0) \cdot P_{0 \mid 0} \cdot \bar{Y}_{0,0}+\operatorname{Pr}(Z=1) \cdot P_{0 \mid 1} \cdot \bar{Y}_{1,0} \\
& +\operatorname{Pr}(Z=1) \cdot P_{0 \mid 0} \cdot \bar{Y}_{0,0}+\operatorname{Pr}(Z=0) \cdot P_{0 \mid 1} \cdot \bar{Y}_{1,0}, \\
& =\operatorname{Pr}(D=0) \cdot E(Y \mid D=0)+\operatorname{Pr}(Z=1) \cdot P_{0 \mid 0} \cdot \bar{Y}_{0,0}+\operatorname{Pr}(Z=0) \cdot P_{0 \mid 1} \cdot \bar{Y}_{1,0},
\end{aligned}
$$

the bounds on $\Delta_{D=1}$ correspond to

$$
\begin{aligned}
\Delta_{D=1}^{U B} & =E(Y \mid D=1)-\frac{\left(P_{1 \mid 0}-\pi_{01}^{\min }\right) \cdot y^{L B}+\operatorname{Pr}(Z=1) \cdot P_{0 \mid 0} \cdot \bar{Y}_{0,0}+\operatorname{Pr}(Z=0) \cdot P_{0 \mid 1} \cdot \bar{Y}_{1,0}}{\operatorname{Pr}(D=1)} \\
& +\frac{\left(P_{0 \mid 1}-\pi_{01}^{\min }\right) \cdot \min \left(\bar{Y}_{0,0}\left(\max \mid q_{0,0}^{00, \pi_{01}^{\min }}\right), \bar{Y}_{1,0}\left(\max \mid q_{1,0}^{00, \pi_{01}^{\min }}\right)\right)}{\operatorname{Pr}(D=1)}, \\
\Delta_{D=1}^{L B} & =E(Y \mid D=1)-\frac{\left(P_{1 \mid 0}-\pi_{01}^{\min }\right) \cdot y^{U B}+\operatorname{Pr}(Z=1) \cdot P_{0 \mid 0} \cdot \bar{Y}_{0,0}+\operatorname{Pr}(Z=0) \cdot P_{0 \mid 1} \cdot \bar{Y}_{1,0}}{\operatorname{Pr}(D=1)} \\
& +\frac{\left(P_{0 \mid 1}-\pi_{01}^{\min }\right) \cdot \max \left(\bar{Y}_{0,0}\left(\min \mid q_{0,0}^{00, \pi_{01}^{\min }}\right), \bar{Y}_{1,0}\left(\min \mid q_{1,0}^{00, \pi_{01}^{\min }}\right)\right)}{\operatorname{Pr}(D=1)} .
\end{aligned}
$$

The bounds of the ATE on the non-treated, $\Delta_{D=0}=E(Y(1)-Y(0) \mid D=0)$, are obtained in a symmetric way. As $E(Y(0) \mid D=0)=E(Y \mid D=0)$ and $E(Y(1) \mid D=$ $0)=\frac{E(Y(1))-\operatorname{Pr}(D=1) \cdot E(Y \mid D=1)}{\operatorname{Pr}(D=0)}$, they are

$$
\begin{aligned}
& \Delta_{D=0}^{U B}=\frac{E(Y(1))^{U B}-\operatorname{Pr}(D=1) \cdot E(Y \mid D=1)}{\operatorname{Pr}(D=0)}-E(Y \mid D=0), \\
& \Delta_{D=0}^{L B}=\frac{E(Y(1))^{L B}-\operatorname{Pr}(D=1) \cdot E(Y \mid D=1)}{\operatorname{Pr}(D=0)}-E(Y \mid D=0) .
\end{aligned}
$$

Furthermore, since 
$\operatorname{Pr}(D=1) \cdot E(Y \mid D=1)=\operatorname{Pr}(Z=0) \cdot P_{1 \mid 0} \cdot \bar{Y}_{0,1}+\operatorname{Pr}(Z=1) \cdot P_{1 \mid 1} \cdot \bar{Y}_{1,1}$

and

$$
\begin{aligned}
P_{1 \mid 1} \cdot \bar{Y}_{1,1}+P_{1 \mid 0} \cdot \bar{Y}_{0,1} & =\operatorname{Pr}(Z=0) \cdot P_{1 \mid 0} \cdot \bar{Y}_{0,1}+\operatorname{Pr}(Z=1) \cdot P_{1 \mid 1} \cdot \bar{Y}_{1,1} \\
& +\operatorname{Pr}(Z=1) \cdot P_{1 \mid 0} \cdot \bar{Y}_{0,1}+\operatorname{Pr}(Z=0) \cdot P_{1 \mid 1} \cdot \bar{Y}_{1,1} \\
& =\operatorname{Pr}(D=1) \cdot E(Y \mid D=1)+\operatorname{Pr}(Z=1) \cdot P_{1 \mid 0} \cdot \bar{Y}_{0,1}+\operatorname{Pr}(Z=0) \cdot P_{1 \mid 1} \cdot \bar{Y}_{1,1},
\end{aligned}
$$

the bounds on $\Delta_{D=0}$ are given by

$$
\begin{aligned}
\Delta_{D=0}^{U B} & =\frac{\left(P_{0 \mid 1}-\pi_{01}^{\min }\right) \cdot y^{U B}+\operatorname{Pr}(Z=1) \cdot P_{1 \mid 0} \cdot \bar{Y}_{1,0}+\operatorname{Pr}(Z=0) \cdot P_{1 \mid 1} \cdot \bar{Y}_{1,1}}{\operatorname{Pr}(D=0)} \\
& -\frac{\left(P_{1 \mid 0}-\pi_{01}^{\min }\right) \cdot \max \left(\bar{Y}_{1,1}\left(\min \mid q_{1,1}^{11, \pi_{01}^{\min }}\right), \bar{Y}_{0,1}\left(\min \mid q_{0,1}^{11, \pi_{01}^{\min }}\right)\right)}{\operatorname{Pr}(D=0)}-E(Y \mid D=0), \\
\Delta_{D=0}^{L B} & =\frac{\left(P_{0 \mid 1}-\pi_{01}^{\min }\right) \cdot y^{L B}+\operatorname{Pr}(Z=1) \cdot P_{1 \mid 0} \cdot \bar{Y}_{1,0}+\operatorname{Pr}(Z=0) \cdot P_{1 \mid 1} \cdot \bar{Y}_{1,1}}{\operatorname{Pr}(D=0)} \\
& -\frac{\left(P_{1 \mid 0}-\pi_{01}^{\min }\right) \cdot \min \left(\bar{Y}_{1,1}\left(\max \mid q_{1,1}^{11, \pi_{01}^{\min }}\right), \bar{Y}_{0,1}\left(\max \mid q_{0,1}^{11, \pi_{01}^{\min }}\right)\right)}{\operatorname{Pr}(D=0)}-E(Y \mid D=0) .
\end{aligned}
$$

Interestingly, the bounds on $\Delta_{D=1}$ and $\Delta_{D=0}$ are always informative despite the fact that either the bounds for the compliers or the defiers and either the bounds for the always takers or the never takers are not informative.

Finally, the bounds for the ATE on the entire population $\Delta=E(Y(1)-Y(0))$ are directly obtained from the bounds on $E(Y(1))$ and $E(Y(0))$ in (9) and (10):

$$
\begin{aligned}
\Delta^{U B} & =E\left(Y(1)^{U B}-Y(0)^{L B}\right) \\
& =P_{1 \mid 0} \cdot \bar{Y}_{0,1}-\left(P_{1 \mid 0}-\pi_{01}^{\min }\right) \cdot y^{L B}+P_{1 \mid 1} \cdot \bar{Y}_{1,1} \\
& -\left(P_{1 \mid 0}-\pi_{01}^{\min }\right) \cdot \max \left(\bar{Y}_{1,1}\left(\min \mid q_{1,1}^{11, \pi_{01}^{\min }}\right), \bar{Y}_{0,1}\left(\min \mid q_{0,1}^{11, \pi_{01}^{\min }}\right)\right) \\
& +\left(P_{0 \mid 1}-\pi_{01}^{\min }\right) \cdot \min \left(\bar{Y}_{0,0}\left(\max \mid q_{0,0}^{00, \pi_{01}^{\min }}\right), \bar{Y}_{1,0}\left(\max \mid q_{1,0}^{00, \pi_{01}^{\min }}\right)\right) \\
& -P_{0 \mid 1} \cdot \bar{Y}_{1,0}+\left(P_{0 \mid 1}-\pi_{01}^{\min }\right) \cdot y^{U B}-P_{0 \mid 0} \cdot \bar{Y}_{0,0},
\end{aligned}
$$

and 


$$
\begin{aligned}
\Delta^{L B} & =E\left(Y(1)^{L B}-Y(0)^{U B}\right) \\
& =P_{1 \mid 0} \cdot \bar{Y}_{0,1}-\left(P_{1 \mid 0}-\pi_{01}^{\min }\right) \cdot y^{U B}+P_{1 \mid 1} \cdot \bar{Y}_{1,1} \\
& -\left(P_{1 \mid 0}-\pi_{01}^{\min }\right) \cdot \min \left(\bar{Y}_{1,1}\left(\max \mid q_{1,1}^{11, \pi_{01}^{\min }}\right), \bar{Y}_{0,1}\left(\max \mid q_{0,1}^{11, \pi_{01}^{\min }}\right)\right) \\
& +\left(P_{0 \mid 1}-\pi_{01}^{\min }\right) \cdot \max \left(\bar{Y}_{0,0}\left(\min \mid q_{0,0}^{00, \pi_{01}^{\min }}\right), \bar{Y}_{1,0}\left(\min \mid q_{1,0}^{00, \pi_{01}^{\min }}\right)\right) \\
& -P_{0 \mid 1} \cdot \bar{Y}_{1,0}+\left(P_{0 \mid 1}-\pi_{01}^{\min }\right) \cdot y^{L B}-P_{0 \mid 0} \cdot \bar{Y}_{0,0}
\end{aligned}
$$

Again, these bounds are always informative no matter whether the bounds on the effect in some of the principal strata are not informative. In online Appendix A.1.8 we show that it is possible to order the bounds on $\Delta_{D=1}, \Delta_{D=0}$, and $\Delta$ with respect to their tightness. In particular, if $\Delta_{D=1}^{U B}<\Delta_{D=0}^{U B}$ (or equivalently, $\Delta_{D=1}^{L B}>\Delta_{D=0}^{L B}$ ), the bounds on $\Delta_{D=1}$ are tighter than those on $\Delta$, which are in turn tighter than those on $\Delta_{D=0}$. The order is reversed if $\Delta_{D=1}^{U B}>\Delta_{D=0}^{U B}$ (or equivalently $\Delta_{D=1}^{L B}<\Delta_{D=0}^{L B}$ ).

Furthermore, note that $\Delta^{L B}, \Delta^{U B}$ might be narrower than the IV bounds derived by Manski (1990). The reason is that we assume mean independence within strata and unconfounded strata proportions (see Assumption 2), whereas Manski imposes the weaker mean independence of the potential outcomes in the entire population: $E(Y(d) \mid Z=1)=E(Y(d) \mid Z=0)$ for $d \in\{0,1\}$. In contrast, our bounds may be wider than those of Kitagawa (2009), who invokes the stronger assumption of full independence of the instrument and the potential treatment states and outcomes. A formal comparison between the various bounds is given in online Appendices A.1.6 and A.1.7.

Without imposing additional restrictions, the bounds derived in this section are likely to be very wide for most populations. Therefore, they are often not helpful for obtaining meaningful results in applications. For this reason we subsequently introduce further assumptions that appear plausible in many empirical problems and might entail considerably tighter bounds.

\subsection{Monotonicity}

This subsection shows how assuming monotonicity of the treatment in the instrument in addition to Assumption 1 and 2 increases identifying power. (Weak) monotonicity of $D$ in $Z$ implies that the treatment state under $Z=1$ is at least as high as under $Z=0$ for all individuals. 


\section{Assumption 3:}

$\operatorname{Pr}(D(1) \geq D(0))=1$ (monotonicity).

As the potential treatment state never decreases in the instrument, the existence of the defiers (stratum 01) is ruled out. A symmetric result is obtained by assuming $\operatorname{Pr}(D(0) \geq D(1))=1$ which implies that stratum 10 does not exist. Note that assuming $\operatorname{Pr}(D(1) \geq D(0))=1$ (positive monotonicity) is only consistent with the data if $P_{1 \mid 1}-P_{1 \mid 0}>0$, otherwise stratum 01 must necessarily exist. Similarly, $\operatorname{Pr}(D(0) \geq D(1))=1$ (negative monotonicity) requires that $P_{1 \mid 0}-P_{1 \mid 1}>0$, see Table 3. Even though these are necessary conditions for the respective monotonicity assumption, they are not sufficient. Due to the symmetry of positive and negative monotonicity, we will only focus on Assumption 3 (positive monotonicity) in the subsequent discussion.

In their seminal paper on the identification of the local average treatment effect (LATE), Imbens and Angrist (1994) (see also Angrist, Imbens, and Rubin, 1996) show that $\Delta_{10}$ is point identified under Assumptions 1, 2, and 3. I.e., the bounds collapse to a single point given that $\pi_{01}$ is equal to zero:

$$
\begin{aligned}
\Delta_{10} & =\left(\frac{P_{1 \mid 1}}{P_{1 \mid 1}-P_{1 \mid 0}} \cdot \bar{Y}_{1,1}-\frac{P_{1 \mid 0}}{P_{1 \mid 1}-P_{1 \mid 0}} \cdot \bar{Y}_{0,1}\right)-\left(\frac{P_{0 \mid 0}}{P_{1 \mid 1}-P_{1 \mid 0}} \cdot \bar{Y}_{0,0}-\frac{P_{0 \mid 1}}{P_{1 \mid 1}-P_{1 \mid 0}} \cdot \bar{Y}_{1,0}\right) \\
& =\frac{\left(P_{1 \mid 1} \cdot \bar{Y}_{1,1}+P_{0 \mid 1} \cdot \bar{Y}_{1,0}\right)-\left(P_{1 \mid 0} \cdot \bar{Y}_{0,1}+P_{0 \mid 0} \cdot \bar{Y}_{0,0}\right)}{P_{1 \mid 1}-P_{1 \mid 0}} \\
& =\frac{\operatorname{Pr}(D=1 \mid Z=1) \cdot E(Y \mid Z=1, D=1)+\operatorname{Pr}(D=0 \mid Z=1) \cdot E(Y \mid Z=1, D=0)}{\operatorname{Pr}(D=1 \mid Z=1)-\operatorname{Pr}(D=1 \mid Z=0)} \\
& -\frac{\operatorname{Pr}(D=1 \mid Z=0) \cdot E(Y \mid Z=0, D=1)+\operatorname{Pr}(D=0 \mid Z=0) \cdot E(Y \mid Z=0, D=0)}{\operatorname{Pr}(D=1 \mid Z=1)-\operatorname{Pr}(D=1 \mid Z=0)} \\
& =\frac{E(Y \mid Z=1)-E(Y \mid Z=0)}{E(D \mid Z=1)-E(D \mid Z=0)}
\end{aligned}
$$

The last equality gives the well known result that the ATE on the compliers is just the ratio of two differences in conditional expectations, namely the intention to treat effect divided by the share of compliers. Under monotonicity, the observed subgroup with $Z=0$ and $D=1$ consists of always takers only and therefore, $\bar{Y}_{0,1}$ immediately gives the mean potential outcome under treatment for the always takers. Thus, an optimization of the kind $\max \left(\bar{Y}_{1,1}\left(\min \mid q_{1,1}^{11}\right), \bar{Y}_{0,1}\right)$ and $\min \left(\bar{Y}_{1,1}\left(\max \mid q_{1,1}^{11}\right), \bar{Y}_{0,1}\right)$ (with $\left.\pi_{01}=0\right)$ as it was used for the bounds in section 3.1 is not required here. Note, however, that this comparison gives a testable implication for the identifying assumptions. 
If it is satisfied, $\bar{Y}_{1,1}\left(\min \mid q_{1,1}^{11}\right) \leq \bar{Y}_{0,1} \leq \bar{Y}_{1,1}\left(\max \mid q_{1,1}^{11}\right)$, otherwise $Z$ has a direct effect on the outcomes of the always takers. Similarly, $\bar{Y}_{1,0}$ is the mean potential outcome under non-treatment for the never takers. Therefore, another testable implication is $\bar{Y}_{0,0}\left(\min \mid q_{0,0}^{00}\right) \leq \bar{Y}_{1,0} \leq \bar{Y}_{0,0}\left(\max \mid q_{0,0}^{00}\right)$. We refer to Huber and Mellace (2013b) for a joint test of these implications.

In the absence of defiers, the bounds for the always takers and never takers $\left(\Delta_{11}\right.$ and $\left.\Delta_{00}\right)$ simplify to

$$
\begin{aligned}
& \Delta_{11}^{U B}=\bar{Y}_{0,1}-y^{L B}, \\
& \Delta_{11}^{L B}=\bar{Y}_{0,1}-y^{U B},
\end{aligned}
$$

and

$$
\begin{aligned}
& \Delta_{00}^{U B}=y^{U B}-\bar{Y}_{1,0}, \\
& \Delta_{00}^{L B}=y^{L B}-\bar{Y}_{1,0} .
\end{aligned}
$$

These bound are sharp because $E(Y \mid D=1, T=11)$ and $E(Y \mid D=0, T=00)$ are now point identified by $\bar{Y}_{0,1}$ and $\bar{Y}_{1,0}$ (if mean independence within strata holds). However, monotonicity does not impose any restrictions on the distributions of $Y \mid D=0, T=11$ and $Y \mid D=1, T=00$ so that the worst case bounds $y^{L B}, y^{U B}$ have to be assumed.

As in the last section, the bounds on the ATEs on the treated $\left(\Delta_{D=1}\right)$, the nontreated $\left(\Delta_{D=0}\right)$, and the entire population $(\Delta)$ can be expressed as functions of the bounds on $E(Y(1))$ and $E(Y(0))$. In the online appendix we show that under monotonicity,

$$
\begin{aligned}
& E(Y(1))^{U B}=P_{0 \mid 1} \cdot y^{U B}+P_{1 \mid 1} \cdot \bar{Y}_{1,1}, \\
& E(Y(1))^{L B}=P_{0 \mid 1} \cdot y^{L B}+P_{1 \mid 1} \cdot \bar{Y}_{1,1}, \\
& E(Y(0))^{U B}=P_{1 \mid 0} \cdot y^{U B}+P_{0 \mid 0} \cdot \bar{Y}_{0,0}, \\
& E(Y(0))^{L B}=P_{1 \mid 0} \cdot y^{L B}+P_{0 \mid 0} \cdot \bar{Y}_{0,0},
\end{aligned}
$$

so that the bounds on the various populations are given by 


$$
\begin{aligned}
& \Delta_{D=1}^{U B}=E(Y \mid D=1)-\frac{P_{1 \mid 0} \cdot y^{L B}+\operatorname{Pr}(Z=1) \cdot\left(P_{0 \mid 0} \cdot \bar{Y}_{0,0}+P_{0 \mid 1} \cdot \bar{Y}_{1,0}\right)}{\operatorname{Pr}(D=1)}, \\
& \Delta_{D=1}^{L B}=E(Y \mid D=1)-\frac{P_{1 \mid 0} \cdot y^{U B}+\operatorname{Pr}(Z=1) \cdot\left(P_{0 \mid 0} \cdot \bar{Y}_{0,0}+P_{0 \mid 1} \cdot \bar{Y}_{1,0}\right)}{\operatorname{Pr}(D=1)}, \\
& \Delta_{D=0}^{U B}=\frac{P_{0 \mid 1} \cdot y^{U B}+\operatorname{Pr}(Z=0) \cdot\left(P_{1 \mid 0} \cdot \bar{Y}_{1,0}+P_{1 \mid 1} \cdot \bar{Y}_{1,1}\right)}{\operatorname{Pr}(D=0)}-E(Y \mid D=0), \\
& \Delta_{D=0}^{L B}=\frac{P_{0 \mid 1} \cdot y^{L B}+\operatorname{Pr}(Z=0) \cdot\left(P_{1 \mid 0} \cdot \bar{Y}_{1,0}+P_{1 \mid 1} \cdot \bar{Y}_{1,1}\right)}{\operatorname{Pr}(D=0)}-E(Y \mid D=0),
\end{aligned}
$$

and

$$
\begin{aligned}
& \Delta^{U B}=P_{0 \mid 1} \cdot y^{U B}+P_{1 \mid 1} \cdot \bar{Y}_{1,1}-P_{1 \mid 0} \cdot y^{L B}-P_{0 \mid 0} \cdot \bar{Y}_{0,0}, \\
& \Delta^{L B}=P_{0 \mid 1} \cdot y^{L B}+P_{1 \mid 1} \cdot \bar{Y}_{1,1}-P_{1 \mid 0} \cdot y^{U B}-P_{0 \mid 0} \cdot \bar{Y}_{0,0} .
\end{aligned}
$$

Balke and Pearl (1997), Heckman and Vytlacil (2001), and Kitagawa (2009) show that under monotonicity, their bounds on the ATE in the entire population coincide with the bounds of Manski (1990), who only invokes mean independence in the entire population. I.e., Assumption 3 does, if it is satisfied, not bring any additional identifying power for $\Delta$. Interestingly, this is also the case for our bounds on the ATEs on the entire population, the treated, and the non-treated. As all these bounds are optimized at $\pi_{01}^{\min }=\max \left(0, P_{1 \mid 0}-P_{1 \mid 1}\right)$ (at least for continuous outcomes), it follows under a satisfaction of monotonicity that $P_{1 \mid 1}-P_{1 \mid 0} \geq 0$ and therefore, $\pi_{01}^{\min }=0$. For this reason, imposing the monotonicity assumption, which amounts to setting $\pi_{01}=\pi_{01}^{\min }$, does not further tighten the bounds if a defier proportion of zero is already a priori consistent with the data.

\subsection{Mean dominance}

Mean dominance or the stronger stochastic dominance assumption have been used in the sample selection framework by Zhang and Rubin (2003), Lechner and Melly (2007), Blundell et al. (2007), Zhang et al. (2008), and Huber and Mellace (2013a). We will show that mean dominance also bears identifying power in the IV framework.

\section{Assumption 4:}

$E[Y(d) \mid T=10] \geq E[Y(d) \mid T=t] \quad \forall d \in\{0,1\}, t \in\{11,00\}$ (mean dominance).

Assumption 4 states that the mean potential outcomes of the compliers under treat- 
ment and non-treatment are at least as high as those of the always and never takers. Note that the particular mean dominance assumption considered here is only one out of many possible relations between the potential outcomes of various strata. Its plausibility has to be judged in the light of the empirical application and theoretical considerations. In Section 5, we present an example where the compliers are likely to have weakly higher mean potential educational outcomes than both the always takers and the never takers due to plausibly being more able and/or motivated on average. As discussed in the next subsection, mean dominance has testable implications if it is jointly assumed with monotonicity. In the application presented in Section 5 we will test Assumption 4 and show that it is not rejected at any conventional significance level.

The bounds on the ATEs in the various principal strata as well as among the treated, non-treated, and entire populations are provided in the online appendix. Under Assumptions 1, 2, and 4, online Appendix A.3.2 outlines the moment inequalities that provide necessary and sufficient conditions for the defiers' proportion $\pi_{01}$ being the identified set, denoted by $\mathcal{P}^{* *}$. The construction of the latter based on linear linear programming is presented in online Appendix A.3.1. Note that in general $\mathcal{P}^{* *} \subseteq \mathcal{P}^{*} \subseteq \mathcal{P}$. Finally, we derive the bounds on the various ATEs as well as the mean potential outcomes in online Appendix A.3.3.

\subsection{Monotonicity and mean dominance}

We subsequently derive the bounds under both monotonicity (Assumption 3) and mean dominance (Assumption 4). Since $\Delta_{10}$ is point identified under Assumptions 1 to 3, Assumption 4 does not bring any further improvement w.r.t. the compliers. For all other populations, the bounds become tighter when invoking both assumptions.

The upper and lower bounds of the ATE on the always takers are now

$$
\begin{aligned}
& \Delta_{11}^{U B}=\bar{Y}_{0,1}-y^{L B} \\
& \Delta_{11}^{L B}=\bar{Y}_{0,1}-\left(\frac{P_{0 \mid 0}}{P_{1 \mid 1}-P_{1 \mid 0}} \cdot \bar{Y}_{0,0}-\frac{P_{0 \mid 1}}{P_{1 \mid 1}-P_{1 \mid 0}} \cdot \bar{Y}_{1,0}\right) .
\end{aligned}
$$

As under mean dominance, the upper bound of the always takers' mean potential outcome under non-treatment cannot be higher than the compliers' upper bound under non-treatment. Furthermore, monotonicity implies that the latter is point identified 
by $\frac{P_{0 \mid 0}}{P_{1 \mid 1}-P_{1 \mid 0}} \cdot \bar{Y}_{0,0}-\frac{P_{0 \mid 1}}{P_{1 \mid 1}-P_{1 \mid 0}} \cdot \bar{Y}_{1,0}$. Again, $\Delta_{11}^{L B}$ is sharp by Lemma 1 in online Appendix A.3. Similarly, the bounds for the never takers tighten to

$$
\begin{aligned}
\Delta_{00}^{U B} & =\left(\frac{P_{1 \mid 1}}{P_{1 \mid 1}-P_{1 \mid 0}} \cdot \bar{Y}_{1,1}-\frac{P_{1 \mid 0}}{P_{1 \mid 1}-P_{1 \mid 0}} \cdot \bar{Y}_{0,1}\right)-\bar{Y}_{1,0}, \\
\Delta_{00}^{L B} & =y^{L B}-\bar{Y}_{1,0} .
\end{aligned}
$$

By the monotonicity assumption, $\frac{P_{1 \mid 1}}{P_{1 \mid 1}-P_{1 \mid 0}} \cdot \bar{Y}_{1,1}-\frac{P_{1 \mid 0}}{P_{1 \mid 1}-P_{1 \mid 0}} \cdot \bar{Y}_{0,1}$ is the compliers' mean potential outcome under treatment. Under mean dominance, this is an upper bound for the never takers' mean potential outcome under treatment.

Under both monotonicity and mean dominance, the upper bounds of $E(Y(0))$ and $E(Y(1))$ become

$$
\begin{aligned}
& E(Y(1))^{U B}=P_{0 \mid 1} \cdot\left(\frac{P_{1 \mid 1} \cdot \bar{Y}_{1,1}-P_{1 \mid 0} \cdot \bar{Y}_{0,1}}{P_{1 \mid 1}-P_{1 \mid 0}}\right)+P_{1 \mid 1} \cdot \bar{Y}_{1,1} \\
& E(Y(0))^{U B}=P_{1 \mid 0} \cdot\left(\frac{P_{0 \mid 0} \cdot \bar{Y}_{0,0}-P_{0 \mid 1} \cdot \bar{Y}_{1,0}}{P_{1 \mid 1}-P_{1 \mid 0}}\right)+P_{0 \mid 0} \cdot \bar{Y}_{0,0}
\end{aligned}
$$

while the lower bounds are equivalent to those under monotonicity alone.

Therefore, the bounds on the ATEs on the treated, non-treated, and the entire population are given by

$$
\begin{aligned}
& \Delta_{D=1}^{U B}=E(Y \mid D=1)-\frac{P_{1 \mid 0} \cdot y^{L B}+\operatorname{Pr}(Z=1) \cdot\left(P_{0 \mid 0} \cdot \bar{Y}_{0,0}+P_{0 \mid 1} \cdot \bar{Y}_{1,0}\right)}{\operatorname{Pr}(D=1)} \\
& \Delta_{D=1}^{L B}=E(Y \mid D=1)-\frac{P_{1 \mid 0} \cdot\left(\frac{P_{0 \mid 0} \cdot \bar{Y}_{0,0}-P_{0 \mid 1} \cdot \bar{Y}_{1,0}}{P_{1 \mid 1}-P_{1 \mid 0}}\right)+\operatorname{Pr}(Z=1) \cdot\left(P_{0 \mid 0} \cdot \bar{Y}_{0,0}+P_{0 \mid 1} \cdot \bar{Y}_{1,0}\right)}{\operatorname{Pr}(D=1)},
\end{aligned}
$$

$$
\begin{aligned}
\Delta_{D=0}^{U B} & =\frac{P_{0 \mid 1} \cdot\left(\frac{P_{1 \mid 1} \cdot \bar{Y}_{1,1}-P_{1 \mid 0} \cdot \bar{Y}_{0,1}}{P_{1 \mid 1}-P_{1 \mid 0}}\right)+\operatorname{Pr}(Z=0) \cdot\left(P_{1 \mid 0} \cdot \bar{Y}_{1,0}+P_{1 \mid 1} \cdot \bar{Y}_{1,1}\right)}{\operatorname{Pr}(D=0)}-E(Y \mid D=0), \\
\Delta_{D=0}^{L B} & =\frac{P_{0 \mid 1} \cdot y^{L B}+\operatorname{Pr}(Z=0) \cdot\left(P_{1 \mid 0} \cdot \bar{Y}_{1,0}+P_{1 \mid 1} \cdot \bar{Y}_{1,1}\right)}{\operatorname{Pr}(D=0)}-E(Y \mid D=0),
\end{aligned}
$$

and

$$
\begin{aligned}
& \Delta^{U B}=P_{0 \mid 1} \cdot\left(\frac{P_{1 \mid 1} \cdot \bar{Y}_{1,1}-P_{1 \mid 0} \cdot \bar{Y}_{0,1}}{P_{1 \mid 1}-P_{1 \mid 0}}\right)+P_{1 \mid 1} \cdot \bar{Y}_{1,1}-P_{1 \mid 0} \cdot y^{L B}-P_{0 \mid 0} \cdot \bar{Y}_{0,0}, \\
& \Delta^{L B}=P_{0 \mid 1} \cdot y^{L B}+P_{1 \mid 1} \cdot \bar{Y}_{1,1}-P_{1 \mid 0} \cdot\left(\frac{P_{0 \mid 0} \cdot \bar{Y}_{0,0}-P_{0 \mid 1} \cdot \bar{Y}_{1,0}}{P_{1 \mid 1}-P_{1 \mid 0}}\right)-P_{0 \mid 0} \cdot \bar{Y}_{0,0} .
\end{aligned}
$$

As a final remark it is worth noting that under Assumptions 1 to 3, Assumption 
4 (mean dominance) has some testable implications. Recall that the always takers' mean potential outcome is identified by $\bar{Y}_{0,1}$. Therefore, mean dominance of the compliers can be tested by comparing $\bar{Y}_{0,1}$ and $\bar{Y}_{1,1}$, which also encounters compliers and, therefore, has to dominate. Equivalently, $\bar{Y}_{1,0}$ is the never takers' mean potential outcome under non-treatment and must be dominated by $\bar{Y}_{0,0}$, which contains never takers and compliers. The intuition is that since the mean potential outcome of the always takers (never takers) is not affected by $Z$ under mean independence within strata, the observed mean outcome consisting of both compliers and always takers (never takers) dominates the observed mean outcome of the always takers (never takers) only. The respective null hypotheses to be tested are $\bar{Y}_{1,1} \geq \bar{Y}_{0,1}$ and $\bar{Y}_{0,0} \geq$ $\bar{Y}_{1,0}$. See Section 5 for an application of mean dominance tests.

\section{Estimation}

Under Assumptions 1 to 3 or 1 to 4 , estimators of the bounds can be constructed by using the sample analogs of the bounds derived under the various assumptions, which is straightforward. To this end, we define the following sample parameters:

$$
\begin{aligned}
\hat{P}_{1 \mid 1}= & \frac{\sum_{i=1}^{n} D_{i} \cdot Z_{i}}{\sum_{i=1}^{n} Z_{i}}, \quad \hat{P}_{0 \mid 1}=1-\frac{\sum_{i=1}^{n} D_{i} \cdot Z_{i}}{\sum_{i=1}^{n} Z_{i}}, \quad \hat{P}_{1 \mid 0}=\frac{\sum_{i=1}^{n} D_{i} \cdot\left(1-Z_{i}\right)}{\sum_{i=1}^{n}\left(1-Z_{i}\right)}, \\
\hat{P}_{0 \mid 0}= & 1-\frac{\sum_{i=1}^{n} D_{i} \cdot\left(1-Z_{i}\right)}{\sum_{i=1}^{n}\left(1-Z_{i}\right)}, \quad \hat{\bar{Y}}_{1,1}=\frac{\sum_{i=1}^{n} Y_{i} \cdot D_{i} \cdot Z_{i}}{\sum_{i=1}^{n} D_{i} \cdot Z_{i}}, \quad \hat{\bar{Y}}_{0,1}=\frac{\sum_{i=1}^{n} Y_{i} \cdot D_{i} \cdot\left(1-Z_{i}\right)}{\sum_{i=1}^{n} D_{i} \cdot\left(1-Z_{i}\right)}, \\
\hat{\bar{Y}}_{1,0}= & \frac{\sum_{i=1}^{n} Y_{i} \cdot\left(1-D_{i}\right) \cdot Z_{i}}{\sum_{i=1}^{n}\left(1-D_{i}\right) \cdot Z_{i}}, \quad \hat{\bar{Y}}_{0,0}=\frac{\sum_{i=1}^{n} Y_{i} \cdot\left(1-D_{i}\right) \cdot\left(1-Z_{i}\right)}{\sum_{i=1}^{n}\left(1-D_{i}\right) \cdot\left(1-Z_{i}\right)}, \\
& \hat{\bar{Y}}_{z, d}\left(\max \mid q_{z, d}^{t}\right)=\frac{\sum_{i=1}^{n} Y_{i} \cdot I\left\{D_{i}=d\right\} \cdot I\left\{Z_{i}=z\right\} \cdot I\left\{Y \geq \hat{y}_{1-q_{z, d}^{t}}\right\}}{\sum_{i=1}^{n} I\left\{D_{i}=d\right\} \cdot I\left\{Z_{i}=z\right\} \cdot I\left\{Y \geq \hat{y}_{1-q_{z, d}^{t}}\right\}}, \\
& \hat{\bar{Y}}_{z, d}\left(\min \mid q_{z, d}^{t}\right)=\frac{\sum_{i=1}^{n} Y_{i} \cdot I\left\{D_{i}=d\right\} \cdot I\left\{Z_{i}=z\right\} \cdot I\left\{Y \leq \hat{y}_{q_{z, d}^{t}}\right\}}{\sum_{i=1}^{n} I\left\{D_{i}=d\right\} \cdot I\left\{Z_{i}=z\right\} \cdot I\left\{Y \leq \hat{y}_{q_{z, d}^{t}}\right\}}, \\
\hat{y}_{q_{z, d}^{t}}= & \min \left\{y: \frac{\sum_{i=1}^{n} D_{i} \cdot Z_{i} \cdot I\left\{Y_{i} \leq y\right\}}{\sum_{i=1}^{n} D_{i} \cdot Z_{i}} \geq q_{z, d}^{t}\right\}, \quad \hat{y}^{L B}=\min (Y), \quad \hat{y}^{U B}=\max (Y),
\end{aligned}
$$

where $I\{\cdot\}$ is the indicator function. Using these expressions instead of the population parameters in the various formulas for the bounds immediately yields feasible estimators. $\sqrt{n}$-consistency and asymptotic normality of these estimators follow immediately from the results of Lee (2009) and its discussion is, therefore, omitted.

Under Assumptions 1 and 2 or 1, 2, and 4, however, estimation is non-standard due to the presence of $\min / \max$ and sup/inf operators. For example, the upper bound on 
the compliers under Assumptions 1 and 2 is constructed in two steps. First, the sharp upper bound given $\pi_{01}$ is obtained as the minimum of the four possible combinations of the pairs $\left(\bar{Y}_{1,1}\left(\min \mid q_{1,1}^{11}\right), \bar{Y}_{0,1}\left(\min \mid q_{0,1}^{11}\right)\right)$ and $\left(\bar{Y}_{0,0}\left(\max \mid q_{0,0}^{00}\right), \bar{Y}_{1,0}\left(\max \mid q_{1,0}^{00}\right)\right)$, which are both functions of $Z$. In the second step, the upper bound is derived by taking the sup of the bound over $\pi_{01}$.

More general, denote by $\Delta_{t}^{L B}\left(\pi_{01}, z, z^{\prime}\right), \Delta_{t}^{U B}\left(\pi_{01}, z, z^{\prime}\right)^{12}$ the upper and lower bounds of any $\Delta_{t}$ conditional on $\pi_{01}, Z=z$ in the first min (max) operator and $Z=z^{\prime}$ in the second one. To simplify the exposition, we define

$$
v=\left\{\begin{array}{ll}
1 & \text { if } z=1, z^{\prime}=1 \\
2 & \text { if } z=1, z^{\prime}=0 \\
3 & \text { if } z=0, z^{\prime}=1 \\
4 & \text { if } z=0, z^{\prime}=0
\end{array} .\right.
$$

This allows rewriting $\Delta_{t}^{L B}\left(\pi_{01}, z, z^{\prime}\right), \Delta_{t}^{U B}\left(\pi_{01}, z, z^{\prime}\right)$ as $\Delta_{t}^{L B}\left(\pi_{01}, v\right), \Delta_{t}^{U B}\left(\pi_{01}, v\right)$. Then, the identification region of $\Delta_{t}$ is obtained by optimizing over admissible values of $\pi_{01} \in \mathcal{P}^{*}$ and $v \in V=\{1,2,3,4\}$ :

$$
\inf _{\pi_{01} \in \mathcal{P}^{*}}\left\{\max _{v \in V}\left[\Delta_{t}^{L B}\left(\pi_{01}, v\right)\right]\right\} \leq \Delta_{t} \leq \sup _{\pi_{01} \in \mathcal{P}^{*}}\left\{\min _{v \in V}\left[\Delta_{t}^{U B}\left(\pi_{01}, v\right)\right]\right\}
$$

Hirano and Porter (2012) show that for parameters that are non-differentiable functionals of the data (such as min/max and sup/inf operators), asymptotically unbiased estimators do not exist. Therefore, the sample analog estimators of $\inf _{\pi_{01} \in \mathcal{P}^{*}}\left\{\max _{v \in V}\left[\Delta_{t}^{L B}\left(\pi_{01}, v\right)\right]\right\}$ and $\sup _{\pi_{01} \in \mathcal{P}^{*}}\left\{\min _{v \in V}\left[\Delta_{t}^{U B}\left(\pi_{01}, v\right)\right]\right\}$ may suffer from substantial bias. Similarly, the usual estimators of the standard deviations of such sample analog estimators, which are either based on asymptotic approximation or the bootstrap, produce confidence regions with incorrect coverage probabilities. However, note that the biases on bounds and confidence intervals induced by optimizing over the defier proportion and the instrument go in opposite directions. Taking the supremum (infimum) of the upper (lower) bounds over $\pi_{01}$ yields overly conservative inference, while optimizing over $Z$ produces bounds and confidence intervals that are too tight. ${ }^{13}$ For this reason, we

\footnotetext{
${ }^{12}$ This section as well as in online Appendix A.7 focuses on bounds that contain two min/max operators. If the bound contains only one $\min (\max )$ operator, these expressions have to be replaced by $\Delta_{t}^{L B}\left(\pi_{01}, z\right)$, $\Delta_{t}^{U B}\left(\pi_{01}, z\right)$. Estimation is then analogous except that $v=z$ and can therefore only take two (rather than four) values.

${ }^{13}$ By optimizing over admissible defiers proportions $\mathcal{P}^{*}\left(\right.$ or $\left.\mathcal{P}^{* *}\right)$ we ignore the fact that $\mathcal{P}^{*}$ is unknown
} 
ignore the first source of bias due to $\pi_{01}$, but account for the second one due to $Z$ by applying the method proposed in Chernozhukov et al. (2013) and also used in Chen and Flores (2012). In this way we obtain conservative (and half-median-unbiased) point estimates and confidence intervals for the bounds. The method is described in online Appendix A.7.

\section{Application}

We apply the methods outlined in the last sections to a school voucher experiment that was conducted within Colombia's "Programa de Ampliación de Cobertura de la Educación Secundaria" (PACES) in order to evaluate the program's impact on the educational achievement of various subpopulations. The PACES program targeted low income families in Colombia and provided more than 125,000 pupils with vouchers covering somewhat more than half the cost of private secondary schooling. Its goals were, among others, to increase net enrollment rates in secondary education and to raise quality compared to a public only educational system, see King et al. (1997). We use a subsample of the data previously analyzed by Angrist et al. (2002) which consists of 1201 pupils in the capital Bogotá whose average age was 12 years when they had applied for private school vouchers in 1995. After randomly (not) being offered a voucher the applicants were re-interviewed in the second half of 1998 to measure the outcome variables of interest such as the highest grade completed and whether grades had to be repeated.

Table 4: Observed strata proportions

\begin{tabular}{l|cc}
\hline \hline Conditional treatment probability & estimate & standard error \\
\hline$P_{1 \mid 1}=\operatorname{Pr}(D=1 \mid Z=1)$ & 0.561 & $(0.020)$ \\
$P_{0 \mid 1}=\operatorname{Pr}(D=0 \mid Z=1)$ & 0.439 & $(0.020)$ \\
$P_{1 \mid 0}=\operatorname{Pr}(D=1 \mid Z=0)$ & 0.056 & $(0.010)$ \\
$P_{0 \mid 0}=\operatorname{Pr}(D=0 \mid Z=0)$ & 0.944 & $(0.010)$ \\
\hline
\end{tabular}

Let $Z$ denote the random assignment indicator, $Y$ a dummy for never repeating a grade or the highest grade completed, respectively, and $D$ whether private schooling was actually received. As shown in Table 4, compliance with the school voucher assignment was not perfect. Only $56.1 \%$ of the 629 pupils offered a school voucher actually went to private schools, while $43.9 \%$ did not. $94.4 \%$ of the 583 pupils

and needs to be estimated in practice. Developing a statistical inference procedure that would account for the sampling distribution of $\mathcal{P}^{*}$ is, however, beyond the scope of the present paper, which focuses on identification. 
that were randomized out did not receive private schooling, but $5.6 \%$ attended private schools anyway. Table 5 reports the estimated bounds on the strata proportions without monotonicity and the respective point estimates under Assumption 3 (monotonicity). In our application, $\mathcal{P}$, the outer bounds on the proportion of defiers based on the distribution of $(D, Z)$ (given by (6)) coincide with the sharp bounds $\mathcal{P}^{*}$ based on the distribution of $(Y, D, Z)$ under mean independence within principal strata, which are obtained by linear programming as discussed in online Appendix A.1.1. They also coincide with the sharp bounds $\mathcal{P}^{* *}$ under the additional assumption of mean dominance, which are presented in online Appendix A.3.1.

Table 5: Estimated (bounds on the) proportions of latent strata

\begin{tabular}{l|cc}
\hline \hline Latent strata & Bounds without monotonicity & Proportions under monotonicity \\
\hline Always takers & {$[0.000,0.056]$} & 0.056 \\
Compliers & {$[0.505,0.561]$} & 0.505 \\
Never takers & {$[0.383,0.439]$} & 0.439 \\
Defiers & {$[0.000,0.056]$} & - \\
\hline
\end{tabular}

We estimate bounds on the ATEs of the compliers, the always takers, the never takers, the treated, and the total population under mean independence within strata, mean dominance, and/or monotonicity. We do not consider defiers, because $\mathcal{P}=\mathcal{P}^{*}$ and $\hat{P}_{1 \mid 1}>\hat{P}_{0 \mid 1}$ imply that the bounds for the defiers are not informative when only invoking mean independence within strata. Furthermore, defiers are ruled out under monotonicity (and under both monotonicity and mean dominance). Note that also the bounds for the always takers are not informative under mean independence within strata alone, because $\hat{P}_{1 \mid 0}<\hat{P}_{0 \mid 1}$ such that the share of always takers is smaller than the share of never takers. However, under monotonicity and/or mean dominance, informative bounds can be obtained for this stratum.

Whenever optimization over the defier proportion is required, ${ }^{14}$ we use an equidistant grid of 100 values between the minimum (0) and maximum (0.056) possible shares. Under mean independence within strata and/or mean dominance (without monotonicity), we apply the Chernozhukov et al. (2013) procedure (see the last section) for estimation and inference (using a nominal significance level of 5\%) using 5000 bootstraps and 200000 simulations. ${ }^{15}$ Under monotonicity (with and without

\footnotetext{
${ }^{14}$ This concerns the compliers and -due to the discreteness of the outcomes- also the treated and the entire population under Assumptions 1 and 2, and all populations considered under Assumptions 1,2, and 4.

${ }^{15}$ We are indebted to Xuan Chen and Carlos Flores for providing us with their Matlab code implementing the Chernozhukov et al. (2013) procedure and for their helpful advice about its implementation. As we have to estimate 100 variance-covariance matrices for each bound when optimizing over
} 
mean dominance), which implies that standard asympotics apply to the bounds, we compute the $95 \%$ confidence intervals for the ATEs (rather than the bounds) based on the method described in Imbens and Manski (2004):

$$
\left(\hat{\Delta}_{t}^{L B}-1.645 \cdot \hat{\sigma}_{t}^{L B}, \hat{\Delta}_{t}^{U B}+1.645 \cdot \hat{\sigma}_{t}^{U B}\right)
$$

where $\hat{\Delta}_{t}^{L B}, \hat{\Delta}_{t}^{U B}$ are the estimated bounds in stratum $t$ and $\hat{\sigma}_{t}^{L B}, \hat{\sigma}_{t}^{U B}$ denote their respective estimated standard errors, ${ }^{16}$ obtained from 5000 bootstrap replications. Concerning the worst case bounds $y^{U B}$ and $y^{L B}$, note that the binary outcome "never repeating a grade" is naturally bounded between 0 and 1 . For the highest grade completed, we take the maximum and minimum values observed in the data, which are 11 and 5 years of schooling, respectively.

Table 6: ATE estimates on "never repeating a grade" and confidence intervals

\begin{tabular}{|c|c|c|c|c|c|}
\hline Assumptions & Compliers & Always takers & Never takers & Treated & Entire pop. \\
\hline Assumptions 1 and 2 only & {$[0.072,0.208]$} & {$[-1.000,1.000]$} & {$[-0.783,0.379]$} & {$[0.044,0.263]$} & {$[-0.261,0.253]$} \\
\hline & $(0.000,0.281)$ & Not informative & $(-0.837,0.426)$ & $(-0.010,0.333)$ & $(-0.302,0.286)$ \\
\hline Mean dominance & {$[0.071,0.207]$} & {$[-0.923,0.966]$} & {$[-0.785,0.350]$} & {$[0.045,0.266]$} & {$[-0.261,0.255]$} \\
\hline & $(0.005,0.250)$ & $(-1.000,0.981)$ & $(-0.811,0.401)$ & $(-0.009,0.333)$ & $(-0.302,0.287)$ \\
\hline Monotonicity & 0.118 & {$[-0.156,0.844]$} & {$[-0.684,0.316]$} & {$[0.070,0.245]$} & {$[-0.249,0.245]$} \\
\hline & $(0.032,0.203)$ & $(-0.263,0.951)$ & $(-0.730,0.363)$ & $(0.010,0.313)$ & $(-0.293,0.277)$ \\
\hline Both & 0.118 & {$[-0.011,0.844]$} & {$[-0.684,0.289]$} & {$[0.095,0.245]$} & {$[-0.241,0.234]$} \\
\hline & $(0.032,0.203)$ & $(-0.138,0.951)$ & $(-0.730,0.340)$ & $(0.025,0.313)$ & $(-0.287,0.270)$ \\
\hline
\end{tabular}

The number of simulations for the half-median-unbiased estimators is 200000

Table 6 presents the results for the outcome "never repeating a grade" after the school voucher assignment under the various assumptions. The bounds of the ATE estimates are given in square brackets, the $95 \%$ confidence intervals are in round brackets. When only invoking Assumptions 1 and 2, the bounds are not informative for the always takers and quite wide for the never takers and the entire population. For the treated, the estimated interval is positive, but the lower bound is not significantly different from zero. For the compliers, the set is significantly positive (on a nominal level of 5\%) and suggests that private schooling decreases the probability to repeat a class by 7 to 21 percentage points. This result suggests that mean independence within strata might have considerable identifying power in applications even when other restrictions such as monotonicity do not appear plausible.

$\pi_{01}$ (one for each value of the grid), some of them are close to being singular. To overcome this problem we use the Matlab function "mchol" by Brian Borchers (downloaded on Feb 06th 2013 from http://infohost.nmt.edu/ ${ }^{\sim}$ borchers/ldlt.html) for regularization.

${ }^{16}$ The confidence intervals apply to cases where the distance between the upper and lower bound of the effect is bounded away from zero, see Stoye (2009). Under point identification (as for the compliers under monotonicity), the conventional two-sided confidence intervals are to be used: $\left(\hat{\Delta}_{t}-1.96 \cdot \hat{\sigma}_{t}, \hat{\Delta}_{t}+1.96 \cdot \hat{\sigma}_{t}\right)$, where $\hat{\Delta}_{t}, \hat{\sigma}_{t}$ denote the point estimate of the effect and the estimated standard error. 
Mean dominance slightly narrows the bounds for the always takers, which are now informative, but all in all, the gains in identification are if anything modest. In contrast, monotonicity of $D$ in $Z$ (such that defiers are ruled out) entails point identification of the ATE on the compliers. The positive and significant estimate implies that grade repetition is reduced by roughly 12 percentage points when attending a private school. Also the identification region of the ATE on the treated, which (from a policy perspective) often represent the most interesting population, is now significantly positive. When invoking both monotonicity and mean dominance, the lower bound for the treated is tightened further. The identification regions for the always takers, never takers, and the entire population shrink somewhat, too, but still include the possibility of a zero effect.

Table 7 shows the estimates for the outcome "highest grade completed". Under Assumptions 1 and 2 alone, the estimated set for the ATE on the compliers positive and almost significant. It suggests that attending a private school increases the highest grade completed on average by 0.15 to 0.56 years for this population. Mean dominance does little to shrink the complier bounds. Under monotonicity, the point estimate suggests that schooling is on average raised by a third of a year. When invoking both assumptions, also the ATE on the treated (between 0.29 and 0.76 years) is significantly positive. Even the identification region for the always takers is larger than zero, but the effect is not significant at the $5 \%$ level. All in all, our results support the conclusion of Angrist et al. (2002) that pupils going to private schools benefited from higher educational attainment. We find economically important positive effects on the likelihood not to repeat grades and on the highest grade completed among the compliers, but also among the treated population. The latter result is particularly relevant, because it suggests that the program increases the outcomes of those actually participating, a group that is most likely of more policy interest than the latent population of compliers.

Table 7: ATE estimates on "highest grade completed" and confidence intervals

\begin{tabular}{|c|c|c|c|c|c|}
\hline Assumptions & Compliers & Always takers & Never takers & Treated & Entire pop. \\
\hline \multirow[t]{2}{*}{ Assumptions 1 and 2 only } & {$[0.149,0.562]$} & {$[-6.000,6.000]$} & {$[-2.512,4.019]$} & {$[-0.353,0.760]$} & {$[-1.026,1.984]$} \\
\hline & $(-0.008,0.721)$ & Not informative & $(-2.627,4.248)$ & $(-0.562,0.952)$ & $(-1.155,2.143)$ \\
\hline \multirow[t]{2}{*}{ Mean dominance } & {$[0.155,0.549]$} & {$[-2.007,3.012]$} & {$[-2.513,1.019]$} & {$[0.037,0.810]$} & {$[-0.866,0.734]$} \\
\hline & $(-0.001,0.640)$ & $(-2.624,3.037)$ & $(-2.576,1.121)$ & $(-0.084,0.988)$ & $(-0.984,0.806)$ \\
\hline \multirow[t]{2}{*}{ Monotonicity } & 0.326 & {$[-3.188,2.813]$} & {$[-2.251,3.749]$} & {$[-0.287,0.760]$} & {$[-1.002,1.968]$} \\
\hline & $(0.126,0.526)$ & $(-3.457,2.967)$ & $(-2.362,3.987)$ & $(-0.506,0.940)$ & $(-1.133,2.127)$ \\
\hline \multirow[t]{2}{*}{ Both } & 0.326 & {$[0.115,2.813]$} & {$[-2.251,0.773]$} & {$[0.289,0.760]$} & {$[-0.818,0.661]$} \\
\hline & $(0.126,0.526)$ & $(-0.112,2.967)$ & $(-2.362,0.889)$ & $(0.122,0.940)$ & $(-0.943,0.746)$ \\
\hline
\end{tabular}


As mentioned in Section 3.4, mean dominance of the compliers' potential outcomes has testable implications if monotonicity holds. We therefore bootstrap the sample analogs of $E[Y(1) \mid T=10]-E[Y(1) \mid T=11]$ and $E[Y(0) \mid T=10]-E[Y(0) \mid T=$ $00]$ (with $E[Y(1) \mid T=10]=\frac{P_{1 \mid 1} \cdot \bar{Y}_{1,1}-P_{1 \mid 0} \cdot \bar{Y}_{0,1}}{P_{1 \mid 1}-P_{1 \mid 0}}, E[Y(0) \mid T=10]=\frac{P_{0 \mid 0} \cdot \bar{Y}_{0,0}-P_{0 \mid 1} \cdot \bar{Y}_{1,0}}{P_{0 \mid 0}-P_{0 \mid 1}}$, $E[Y(1) \mid T=11]=\bar{Y}_{0,1}$, and $\left.E[Y(0) \mid T=00]=\bar{Y}_{1,0}\right)$ to test whether the respective mean potential outcome of the compliers dominates that of the always takers under treatment and that of the never takers under non-treatment. ${ }^{17}$ Table 8 reports the mean potential outcomes of the various populations and the p-values of the tests. The results strongly support the mean dominance of the compliers over the always takers under treatment and the mean dominance of the compliers over the never takers under non-treatment.

However, strictly speaking we also have to test whether the compliers dominate the always takers under non-treatment and the never takers under treatment, respectively. Even though this is infeasible (because always takers are never observed under nontreatment just as never takers under treatment), the mean potential outcomes provide indirect evidence that these assumptions are most likely satisfied. First of all, the hypothesis that the mean potential outcome of the compliers under non-treatment dominates the mean potential outcome of the always takers under treatment cannot be rejected for either outcome. I.e., if the ATE on the always takers is either positive or at least not negative by a sufficiently large amount, the mean potential outcome of the always takers under non-treatment cannot be larger than that of the compliers. Furthermore, the never takers can only have a higher mean potential outcome under treatment than the compliers if the ATE on the former is substantially larger than that on the latter (as the mean potential outcome of the never takers under non-treatment is considerably lower than that of the compliers for both outcome variables). In this case, however, it seems irrational of the never takers not to take the treatment such that this scenario appears unlikely.

Given the results of the tests, the question arises under which circumstances it seems plausible that the compliers' mean educational achievement dominates those of

\footnotetext{
${ }^{17}$ Of course, this approach tests mean dominance conditional on the satisfaction of Assumptions 2 and 3 and is otherwise a joint test of all three assumptions. Huber and Mellace (2013b) suggest tests (i) for Assumptions 2 and 3 alone and (ii) (as also Kitagawa, 2013) for full independence of the instrument and potential treatments/outcomes and Assumption 3. For the outcome "never repeating a grade", using the method of Chen and Szroeter (2014) (with a normal smoothing function and $\sqrt{n / 2 \log (\log (n))}$ as tuning parameter for the selection of binding moments) to test (i) and (ii) (with two equidistant probability measures) yields p-values of 1.000 and 0.999, respectively. For "highest grade completed", the respective p-values are 0.993 and 0.851 . Therefore, our data provide no evidence for a violation of (i) or (ii).
} 
Table 8: Mean potential outcomes and mean dominance tests

\begin{tabular}{r|cc}
\hline \hline & never repeating a grade & highest grade completed \\
\hline$E[Y(1) \mid T=10]$ & 0.973 & 8.024 \\
$E[Y(0) \mid T=10]$ & 0.855 & 7.698 \\
$E[Y(1) \mid T=11]$ & 0.844 & 7.813 \\
$E[Y(0) \mid T=00]$ & 0.684 & 7.251 \\
p-value for $H_{0}: E[Y(1) \mid T=10] \geq E[Y(1) \mid T=11]$ & 0.964 & 0.975 \\
p-value for $H_{0}: E[Y(0) \mid T=10] \geq E[Y(0) \mid T=00]$ & 0.997 & 0.998 \\
p-value for $H_{0}: E[Y(0) \mid T=10] \geq E[Y(1) \mid T=11]$ & 0.559 & 0.211 \\
\hline
\end{tabular}

the always and never takers. Suppose that the private schooling decision is a function of (monetary and non-monetary) costs and utility coming from educational achievement. Economic theory suggests that rational households should send their children to private schools only if the expected utility is at least as high as the costs. Always taker households may get a relatively higher utility from education, e.g., because the parents are themselves better educated and, therefore, appreciate education more than the compliers. Furthermore, they may represent the more wealthy households (as they send their children to private schools even without vouchers) such that their relative costs for schooling are lower. This might again be correlated with parental education. Both increased utility and lower relative costs will give relatively more pupils with lower potential outcomes - related to lower ability and/or motivation- the chance to receive private schooling. This line of argumentation is supported by the data, which also contain information on father's and mother's education and the possession of phone, which may be regarded as a proxy for wealth. The means of these variables (which were measured before the assignment) are higher among always takers than among compliers and the differences are significant at the $10 \%$ level. ${ }^{18}$

In contrast, mean parental education and possessing a phone does not significantly differ between the never takers and compliers. Given that they face similar utilities (for a particular level of education) and relative costs as the compliers, it is plausible that the never taker households did not respond to the vouchers because their kids were probably less motivated and/or able and for this reason their expected returns to private schooling were too small. This suggests that the never takers' ATE (and the mean potential outcomes) is lower than those of the compliers, as never taker households were not even willing to pay less than half of the cost of private schooling (recall that the vouchers did not cover the entire expenses).

\footnotetext{
${ }^{18}$ The test statistics are available from the authors upon request.
} 


\section{Conclusion}

This paper sheds light on the question of what can be learnt about the average treatment effects (ATE) on various populations under endogeneity/noncompliance when a valid instrumental variable (IV) is at hand that satisfies mean independence within strata and ignorable assignment. Since the work by Imbens and Angrist (1994) it is well known that a local ATE (LATE) on the compliers (who take the treatment if instrumented, but do not otherwise) is point identified under monotonicity of the treatment in the instrument. Even though point identification is not feasible for other groups, we show that informative bounds can be obtained for the always takers (treated irrespective of the instrument), the never takers (not treated irrespective of the instrument), the treated, the non-treated, and the entire population. We also investigate the identifying power of mean dominance of the potential outcomes of the compliers over those of the always takers and never takers.

The main contribution is the derivation of sharp bounds on the ATE of various populations under monotonicity, mean dominance, and under both assumptions. We also present an application to Colombia's "Programa de Ampliación de Cobertura de la Educación Secundaria", which provided pupils from low income families with vouchers for private secondary schooling, using experimental data previously analyzed by Angrist et al. (2002). We find (on top of the complier effect) a significantly positive ATE on the educational achievement of the treated population, a group of major policy interest. As valuable "by-products" of our identification results we also obtain testable implications of the validity of the instrument and of mean dominance, respectively.

\section{References}

Angrist J, Bettinger E, Bloom E, King E, Kremer M. 2002. Vouchers for private schooling in colombia: Evidence from a randomized natural experiment. The American Economic Review 92: 1535-1558.

Angrist J, Imbens G, Rubin D. 1996. Identification of causal effects using instrumental variables. Journal of American Statistical Association 91: 444-472 (with discussion).

Balke A, Pearl J. 1997. Bounds on treatment effects from studies with imperfect compliance. Journal of the American Statistical Association 92: 1171-1176.

Bhattacharya J, Shaikh AM, Vytlacil E. 2008. Treatment effect bounds under monotonicity assumptions: An application to swan-ganz catheterization. The American Economic Review : 351-356.

Bloom HS. 1984. Accounting for no-shows in experimental evaluation designs. Evaluation Review 8: 225-246. 
Blundell R, Gosling A, Ichimura H, Meghir C. 2007. Changes in the distribution of male and female wages accounting for employment composition using bounds. Econometrica 75: 323-363.

Chen LY, Szroeter J. 2014. Testing multiple inequality hypotheses: a smoothed indicator approach. Journal of Econometrics 178: 678-693.

Chen X, Flores CA. 2012. Bounds on treatment effects in the presence of sample selection and noncompliance: The wage effects of job corps. Working Paper .

Cheng J, Small DS. 2006. Bounds on causal effects in three-arm trials with noncompliance. Journal of the Royal Statistical Society: Series B (Statistical Methodology) 68.

Chernozhukov V, Lee S, Rosen A. 2013. Intersection bounds: Estimation and inference. Econometrica 81: 667-737.

Chiburis RC. 2010. Semiparametric bounds on treatment effects. Journal of Econometrics $\mathbf{1 5 9 .}$

Dawid AP. 2003. Causal inference using influence diagrams: The problem of partial compliance (with discussion). In Green PJ, Hjort NL, Richardson S (eds.) Highly Structured Stochastic Systems. Oxford University Press.

Deaton AS. 2010. Instruments, randomization, and learning about development. Journal of Economic Literature 48: 424-455.

Flores CA, Flores-Lagunes A. 2013. Partial identification of local average treatment effects with an invalid instrument. Journal of Business and Economic Statistics 31: $534-545$.

Frangakis CE, Rubin DB. 2002. Principal stratification in causal inference. Biometrics 58: $21-29$.

Frölich M. 2007. Nonparametric iv estimation of local average treatment effects with covariates. Journal of Econometrics 139: 35-75.

Frölich M, Lechner M. 2010. Combining matching and nonparametric iv estimation: Theory and an application to the evaluation of active labour market policies. University of St Gallen, Department of Economics Discussion Paper No. 2010-21 .

Heckman JJ, LaLonde R, Smith J. 1999. The economics and econometrics of active labour market programs. In Ashenfelter O, Card D (eds.) The Handbook of Labor Economics. New York: North-Holland, 1865-2097.

Heckman JJ, Urzúa S. 2010. Comparing iv with structural models: What simple iv can and cannot identify. Journal of Econometrics 156: 27-37.

Heckman JJ, Vytlacil E. 2001. Instrumental variables, selection models, and tight bounds on the average treatment effects. In Lechner M, Pfeiffer M (eds.) Econometric Evaluation of Labour Market Policies. New York: Center for European Economic Research, 1-15. 
Hirano K, Porter JR. 2012. Impossibility results for nondifferentiable functionals. Econometrica 80: 1769-1790.

Horowitz JL, Manski CF. 1995. Identification and robustness with contaminated and corrupted data. Econometrica 63: 281-302.

Huber M, Mellace G. 2013a. Sharp bounds on causal effects under sample selection. forthcoming in the Oxford Bulletin of Economics and Statistics .

Huber M, Mellace G. 2013b. Testing instrument validity for late identification based on inequality moment constraints. forthcoming in the Review of Economics and Statistics .

Imbens GW. 2004. Nonparametric estimation of average treatment effects under exogeneity: a review. The Review of Economics and Statistics 86: 4-29.

Imbens GW. 2009. Better late than nothing: Some comments on deaton (2009) and heckman and urzua (2009). NBER Working Paper No. 14896 .

Imbens GW, Angrist J. 1994. Identification and estimation of local average treatment effects. Econometrica 62: 467-475.

Imbens GW, Manski CF. 2004. Confidence intervals for partially identified parameters. Econometrica 72: 1845-1857.

King E, Rawlings L, Gutierrez M, Pardo C, Torres C. 1997. Colombia's targeted education voucher program: Features, coverage and participation. Working Paper No. 3, Series on Impact Evaluation of Education Reforms, Development Economics Research Group, The World Bank .

Kitagawa T. 2009. Identification region of the potential outcome distribution under instrument independence. CeMMAP working paper 30/09 .

Kitagawa T. 2014. A test for instrument validity. CeMMAP working paper 34/14 .

Lafférs L. 2013. A note on bounding average treatment effects. Economics Letters 120: $424-428$.

Lechner M, Melly B. 2007. Earnings effects of training programs. IZA Discussion Paper no. 2926 .

Lee DS. 2009. Training, wages, and sample selection: Estimating sharp bounds on treatment effects. Review of Economic Studies 76: 1071-1102.

Manski CF. 1989. Anatomy of the selection problem. The Journal of Human Resources 24: $343-360$.

Manski CF. 1990. Nonparametric bounds on treatment effects. American Economic Review, Papers and Proceedings 80: 319-323.

Manski CF. 1994. The selection problem. In Sims C (ed.) Advances in Econometrics: Sixth World Congress. Cambridge University Press, 143-170. 
Richardson TS, Robins JM. 2010. Analysis of the binary instrumental variable model. In Dechter R, Geffner H, Halpern JY (eds.) Heuristics, probability and causality: a tribute to Judea Pearl. London, UK: College Publications, 415-440.

Robins J. 1989. The analysis of randomized and non-randomizedaids treatment trials using a new approach to causal inference in longitudinal studies. In Sechrest L, Freeman H, Mulley A (eds.) Health Service Research Methodology: A Focus on AIDS. Washington, DC: U.S. Public Health Service, 113-159.

Rubin DB. 1974. Estimating causal effects of treatments in randomized and nonrandomized studies. Journal of Educational Psychology 66: 688-701.

Rubin DB. 1990. Formal modes of statistical inference for causal effects. Journal of Statistical Planning and Inference 25: 279-292.

Shaikh AM, Vytlacil EJ. 2011. Partial identification in triangular systems of equations with binary dependent variables. Econometrica 79: 949-955.

Stoye J. 2009. More on confidence intervals for partially identified parameters. Econometrica 77: 1299-1315.

Vytlacil E. 2002. Independence, monotonicity, and latent index models: An equivalence result. Econometrica 70: 331-341.

Zhang J, Rubin DB. 2003. Estimation of causal effects via principal stratification when some outcome are truncated by death. Journal of Educational and Behavioral Statistics 28: 353-368.

Zhang J, Rubin DB, Mealli F. 2008. Evaluating the effects of job training programs on wages through principal stratification. In Millimet D, Smith J, Vytlacil E (eds.) Advances in Econometrics: Modelling and Evaluating Treatment Effects in Econometrics, volume 21. Elsevier Science Ltd., 117-145. 